Check for updates

Cite this: New J. Chem., 2019, 43,8835

Received 17th March 2019, Accepted 29th April 2019

DOI: 10.1039/c9nj01402k

rsc.li/njc

\title{
Photoacoustic imaging and photothermal therapy in the second near-infrared window
}

\author{
Xiaoguang Ge, $\dagger^{\mathrm{ab}}$ Qinrui Fu, $\dagger^{\mathrm{b}}$ Lin Bai, $\dagger^{\mathrm{a}}$ Bin Chen, ${ }^{\mathrm{a}}$ Renjie Wang, ${ }^{\mathrm{a}}$ Shi Gao*a and \\ Jibin Song (D) *b
}

\begin{abstract}
The second near-infrared (NIR-II) window, with a broadband absorption ranging from $1000 \mathrm{~nm}$ to $1350 \mathrm{~nm}$, has received increasing attention for photoacoustic imaging and imaging-guided NIR-II photothermal therapy. Compared to conventional nanoagents in the first near-infrared (NIR-I) window, with an optical absorption of 700-1000 nm, NIR-II nanoagents offer various merits such as high-performance photothermal treatment outcomes and photoacoustic imaging with both deeper tissue penetration and higher SNR. Thus, this review focuses on the recent progress in photoacoustic imaging and photothermal therapy in the NIR-II window. The merits of the NIR-II window relative to the NIR-I window are systematically compared. This is followed by a summary of nanoagents in the NIR-II window and their biomedical application, including photoacoustic imaging in the NIR-II window and imaging-guided NIR-II photothermal therapy. Finally, the prospects and challenges of both photoacoustic imaging and photothermal therapy in the NIR-II window are summarized.
\end{abstract}

\section{Introduction}

Photoacoustic imaging (PAI) is a promising biomedical molecular imaging technology that depends on the conversion of photons via the photoacoustic (PA) effect and their absorption

${ }^{a}$ Department of Nuclear Medicine, China-Japan Union Hospital of Jilin University, Changchun, Jilin, 130033, China. E-mail: gaoshi@jlu.edu.cn

${ }^{b}$ MOE Key Laboratory for Analytical Science of Food Safety and Biology,

College of Chemistry, Fuzhou University, Fuzhou 350108, China.

E-mail: jibinsong@fzu.edu.cn

$\dagger$ These authors contributed equally to this work.

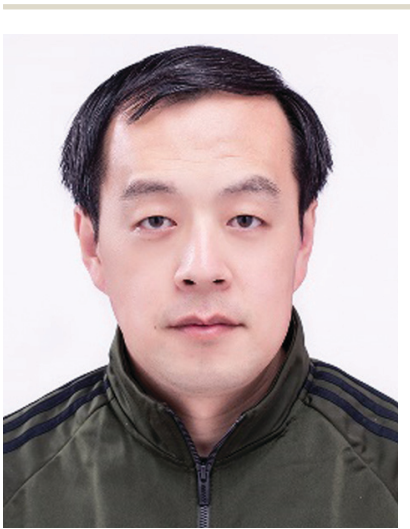

Jibin Song
Jibin Song obtained his PhD degree in Chemical and Biomedical Engineering at Nanyang Technological University, Singapore, in 2014. He then worked with Prof. Xiaoyuan (Shawn) Chen as a Postdoctoral Fellow at the National Institutes of Health (NIH). After finishing his postdoctoral training, he joined the Fuzhou University as a "Min Jiang Scholar" Professor of Analytical Chemistry. Prof. Song has published over 50 papers in high impact journal. His research focuses on developing molecular imaging nanoprobes for bioimaging, biosensing and drug/gene delivery. by exogenous and endogenous contrast agents. This technology combines the excellent spatial resolution of ultrasonic imaging with the exceptional contrast of optical imaging. ${ }^{1-6}$ In recent years, PAI has attracted significant attention due to its high imaging contrast and resolution, low scattering and dissipation in biological tissue, and excellent penetrability and sensitivity to tissue functional characteristics. Therefore, PAI plays an important role in life sciences and cancer therapy., ${ }^{4,7-14}$ Recently, research on PAI has mainly focused on the NIR-I window (700-1000 $\mathrm{nm}$ ); however, due to the strong scattering and absorption of biological tissues in this region, the depth of tissue penetration and the signal-to-noise ratio (SNR) are relatively low. Moreover, the major constituents of biological tissues such as deoxyhemoglobin, oxyhemoglobin, skin, and fat show a decreasing trend of laser absorption in the NIR-II window (1000-1350 nm) ${ }^{15,16}$ (Fig. 1a and b). ${ }^{17,18}$ In addition, The NIR-II window with its longer wavelength excitation laser has the merits of better tolerance of biological tissue, much higher maximum permissible exposure (MPE) in the NIR-II window compared to that of the NIR-I window due to the lower tissue absorption and lower energy of photons at longer wavelengths. ${ }^{18-20}$ For example, $1064 \mathrm{~nm}$ light possesses an MPE value of $1.0 \mathrm{~W} \mathrm{~cm}^{-2}$, while $808 \mathrm{~nm}$ light has a lower MPE value of $0.33 \mathrm{~W} \mathrm{~cm}^{-2}$ according to ANSI Z136.1-2007. ${ }^{16}$ Therefore, the higher MPE of an NIR-II window laser with stronger irradiation power can be used for deep-tissue imaging. Nevertheless, PAI in the NIR-II window has rarely been investigated to date. Reports on PAI in the NIR-II window have been limited to noble metals, ${ }^{21,22}$ copper sulfide nanoparticles, ${ }^{23}$ perfluorocarbon nanodroplets, ${ }^{24}$ 
(a)

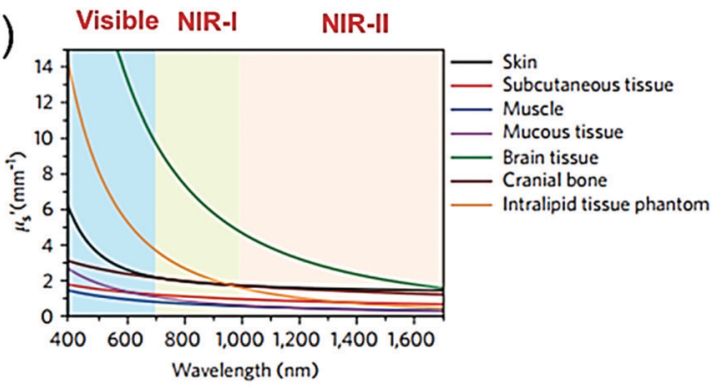

(b)

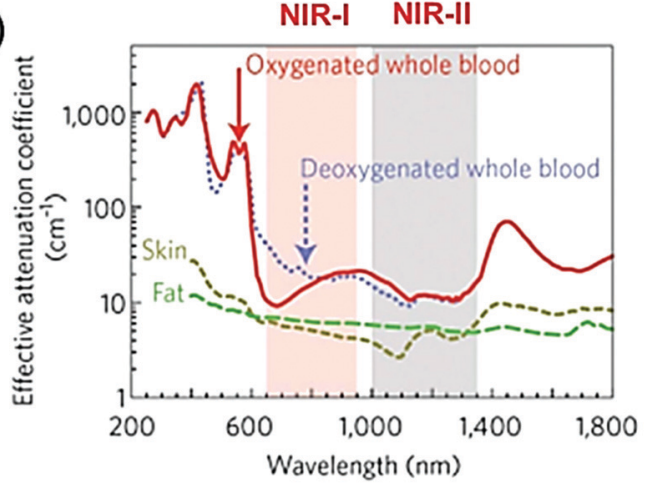

Fig. 1 Visible, NIR-I, and NIR-II windows. (a) Reduced scattering coefficients $\left(\mu_{\mathrm{s}}{ }^{\prime}\right)$ of various biological tissues as a function of wavelength. (b) Effective attenuation coefficients of fat, skin, and blood as a function of wavelength. ( $a$ and b) Reproduced from ref. 17 and 18 with permission from the Nature Publishing Group, Copyright 2017 and 2009, respectively.

phosphorus phthalocyanine, ${ }^{25}$ and semiconducting polymer nanoparticles (SPN). ${ }^{15,26}$ In summary, the NIR-II window possesses intrinsic merits of deeper tissue penetration and higher MPE due to the decreased tissue scattering and the decrease in the energy of long wavelength photons, respectively. Compared to the NIR-I window, the NIR-II window has been reported to offer excellent spatial resolution and enhanced signal-to-background ratio (SBR) for PAI.

Photothermal therapy (PTT) is a promising and non-invasive method for cancer treatment that has advanced to clinical trials. As an effective approach against cancer, PTT has attracted much attention in recent years due to its localized ablation of solid tumors and minimal heating damage to normal tissue adjacent to cancer tissue. The mechanism of PTT involves the adequate accumulation of photothermal (PT) agents within tumors, which then absorb near infrared (NIR) light and convert it into thermal energy to ablate localized tumor cells. ${ }^{27-34}$ Currently, numerous materials have been employed as effective PT agents such as NIR dye, ${ }^{35,36}$ polymer nanoparticles, ${ }^{37,38}$ inorganic nanomaterials, ${ }^{39-41}$ and organic nanomaterials. ${ }^{42-44}$ However, despite their excellent PT effect, the strong background signal, short tissue penetration, and low SNR of NIR-I window nanomaterials still limit their further application in imaging-guided cancer treatment. However, biological applications that use NIR-II laser achieve deeper penetration and larger MPE compared to traditional applications that use the NIR-I laser; thus, PTT in the NIR-II window can improve treatment outcomes. ${ }^{45}$

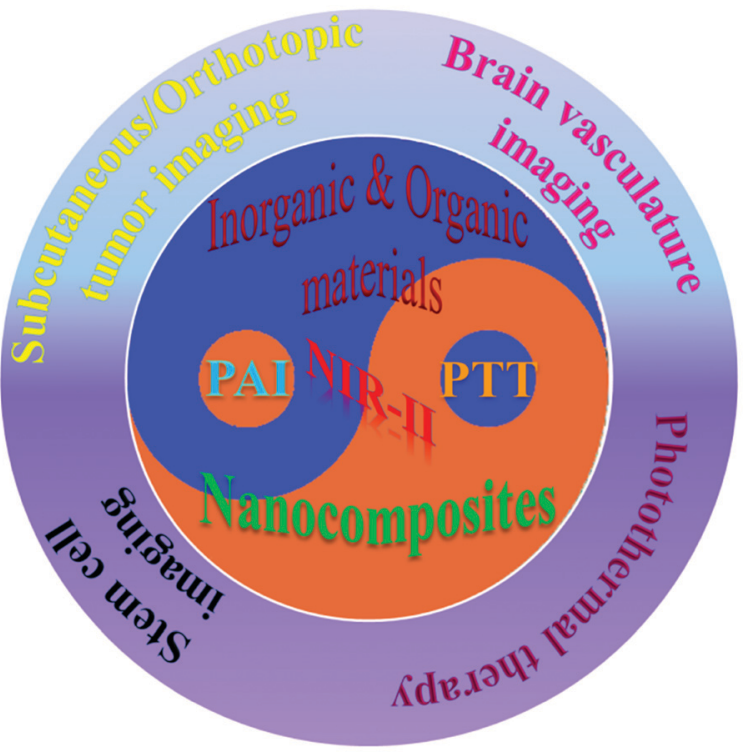

Scheme 1 Schematic illustration of PA imaging contrast agents and their biomedical applications in the NIR-II window.

This review summarizes the recent progress of PA contrast agents and PT agents in the NIR-II window and their biomedical applications (Scheme 1). First, a comprehensive summary and discussion are presented on the available PA contrast agents and PT agents in the NIR-II window, including inorganic materials, organic materials, and other nanocomposites. These NIR-II nanoagents achieve high-performance PT treatment outcomes and allow PA imaging with deeper tissue penetration and higher SNR. Then, we review and compare the effects of PAI and PTT between the NIR-I and NIR-II windows. Subsequently, the recent progress in biomedical applications of these nanoagents is presented, including subcutaneous tumor imaging, deep brain vasculature imaging, orthotopic tumor imaging, stem cell imaging, and imaging-guided NIR-II PTT. Finally, a brief summary is provided together with a discussion of the current opportunities and challenges of NIR-II nanoagents.

\section{PA contrast agents/PT agents in the second near-infrared window}

Nanoagents with excellent absorption in the NIR-II window and outstanding photothermal conversion efficiency can significantly improve the outcomes of cancer treatment and the achieved imaging contrast; therefore, they have aroused significant interest in research. Herein, three categories of NIR-II PA contrast agents and PT agents are reviewed: (1) inorganic materials, (2) organic materials, and (3) other nanomaterials.

\section{Inorganic materials}

Gold nanoparticles (AuNPs) show remarkable potential as PAI contrast agents and PT agents due to their superior light absorption abilities and tunable localized surface plasmon resonance (LSPR) properties. For example, Duan et al. synthesized 
(a)

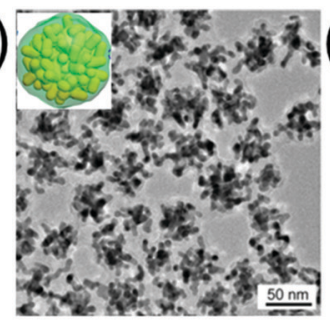

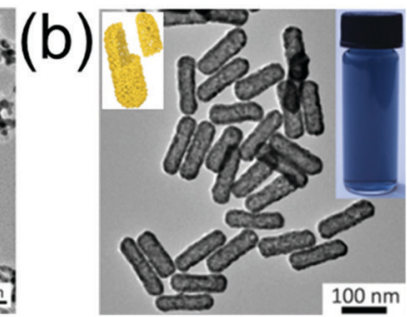

(c)

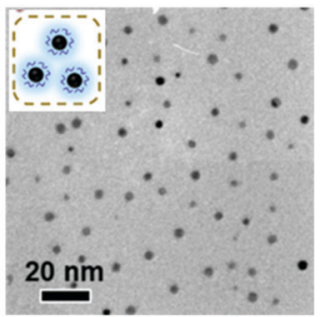

(d)

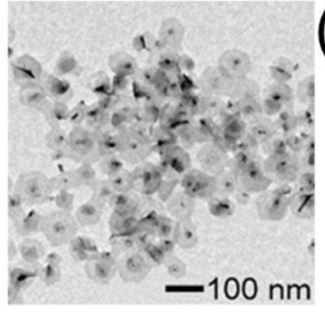

(e)

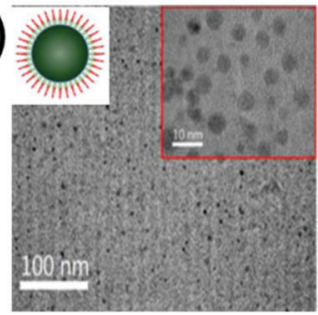

(f)

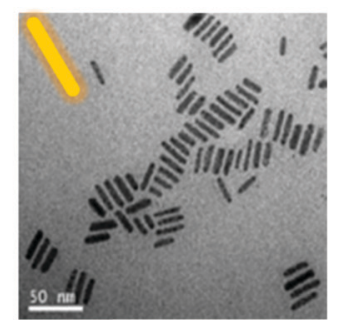

(g)

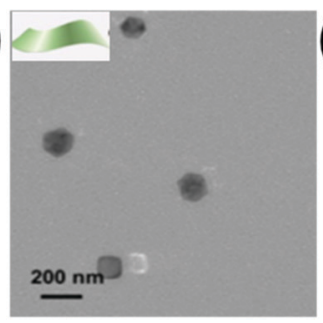

(h)

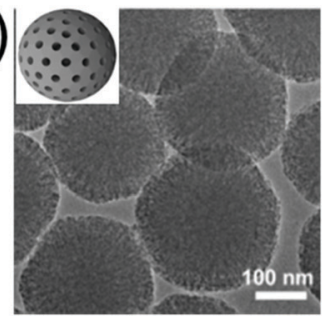

(i)

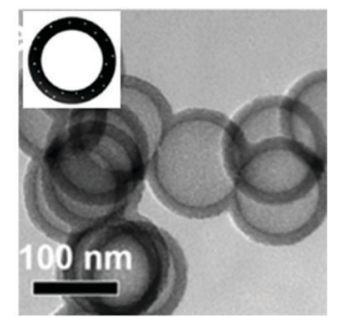

Fig. 2 TEM of (a) hyperbranched Au plasmonic blackbodies (AuPBs), (b) gold hollow nanorods (AuHNRs), (c) bismuth nanoparticles (Bi NPs), (d) Bi $2 \mathrm{Se}_{3}$ nanoplates (NPs), (e) CuS@BSA-RGD NPs, (f) $\mathrm{Cu}_{3} \mathrm{BiS}_{3}$ nanorods (NRs), (g) $\mathrm{Cu}_{2} \mathrm{MnS}_{2} \mathrm{NPs}$, (h) mesoporous carbon nanospheres (Meso-CNs), and (i) black hollow silicon oxide nanoparticles ( $\left.\mathrm{H}-\mathrm{SiO}_{x} \mathrm{NPs}\right)$. (a) was reproduced from ref. 16 with permission from the American Chemical Society, Copyright 2018 , (b) was reproduced from ref. 47 with permission from the American Chemical Society, Copyright 2018, (c) was reproduced from ref. 48 with permission from the American Chemical Society, Copyright 2017, (d) was reproduced from ref. 49 with permission from the Royal Society Chemistry, Copyright 2018, (e) was reproduced from ref. 50 with permission from the Royal Society Chemistry, Copyright 2018, (f) was reproduced from ref. 51 with permission from Elsevier Ltd, Copyright 2017, (g) was reproduced from ref. 52 with permission from Ivyspring International Publisher, Copyright 2017, (h) was reproduced from ref. 53 with permission from Ivyspring International Publisher, Copyright 2018 and (i) was reproduced from ref. 54 with permission from Elsevier Ltd, Copyright 2017.

hyperbranched gold plasmonic blackbodies (AuPBs, Fig. 2a) with a sub-50 nm size via a surfactant-free and seedless method using mussel-inspired dopamine. ${ }^{16}$ AuPBs have wide-band absorption ranging from $400 \mathrm{~nm}$ to $1350 \mathrm{~nm}$ with outstanding photothermal efficiency above $80 \%$ in both the NIR-I and NIR-II windows due to the strong plasmonic coupling among the gold branches. Therefore, AuPBs can be used as PAI contrast agent and for PAI-guided PTT. Gold nanorods (AuNRs) are well-known nanoagents. NIR-II window-responsive PAI contrast agents and PTT agents with sizes exceeding the $200 \mathrm{~nm}$ of traditional AuNRs have been employed; however, their biological safety needs to be further explored. AuNRs with sub-100 nm sizes and tunable NIR-II window plasmonic peaks are promising candidates for in vivo bioapplication and offer excellent biosafety due to their merits in size. For example, Yeh et al. developed AuNRs with a rod-in-shell configuration, which were smaller than $100 \mathrm{~nm}^{46}$ and exhibited broad-band absorption across 350-1350 nm. Both in vitro and in vivo tests showed good biosafety and excellent efficacy in the NIR photothermal ablation of cancer cells when illuminated using a $1064 \mathrm{~nm}$ (NIR-II window) or a $808 \mathrm{~nm}$ (NIR-I window) laser. Zhai et al. developed gold hollow nanorods with adjustable aspect ratios via a templated method with Se-doped Te nanorods, aided by L-cysteine (Fig. 2b). ${ }^{47}$ They had an LSPR peak in the NIR-II window and showed an excellent effect for the chemo-photothermal combined therapy of cancer. A semimetal is a material with properties between a metal and a non-metal. Its appearance shows the properties of a metal, but its chemical properties exhibit both metallic and non-metallic characteristics. After a semi-metallic material is irradiated by infrared light, electron energy is rapidly excited from the valence band to the conduction band, which results in a change in electrical conductivity; thus, detecting infrared light. Bismuth (Bi) is a representative semimetal element with a small carrier effective mass, small band overlap energy, and long Fermi wavelength. When Bi semimetal is transformed into a semiconductor at the nanoscale due to nanoconfinement effects, the applications of Bi-based nanomaterials can be broadened to the biomedicine field as cancer therapeutic agents. ${ }^{37}$ For example, Li et al. prepared ultra-small semimetal Bi nanoparticles with high photostability (Fig. 2c), excellent absorption ability, and outstanding photothermal conversion efficiency in the NIR-II window. Therefore, Bi nanoparticles are suitable for PAI-guided NIR-II window PTT cancer treatment. ${ }^{48}$ $\mathrm{Bi}_{2} \mathrm{Se}_{3}$ has received considerable attention in the biomedical 
field due to its low toxicity, outstanding photothermal conversion efficiency, excellent PA signal, and fast clearance. Kim et al. developed poly(vinyl pyrrolidone) (PVP)-capped $\mathrm{Bi}_{2} \mathrm{Se}_{3}$ nanoplates with strong absorption ability in the NIR-II window, which could be used as PA contrast agents in the NIR-II window for deep tissue PAI (Fig. 2d). ${ }^{49}$ An in vitro experiment showed that an imaging depth up to $4.6 \mathrm{~cm}$, and an in vivo experiment verified the clinical feasibility of $\mathrm{Bi}_{2} \mathrm{Se}_{3}$ nanoagents by PAI of the bladder, gastrointestinal tract, and sentinel lymph nodes in mice. Copper sulphide (CuS)-based nanoparticles are promising nanoagents due to their negligible toxicity, excellent NIR-II absorption, and efficient biodegradability. Li et al. developed CuS semiconductor nanoparticles for NIR-II window PAI of orthotopic brain tumors and rat lymph nodes in deep regions. ${ }^{23}$ Yan $e t$ al. developed tumor targeted protein-CuS nanoparticles through bovine serum albumin (BSA) encapsulated cyclic arginine-glycine-aspartic acid (cRGD) peptide-conjugated CuS nanoparticles (CuS@BSA-RGD NPs) for PAI of orthotopic hepatocellular carcinoma with a high SNR (Fig. 2e).$^{50}$ Copper bismuth sulfide $\left(\mathrm{Cu}_{3} \mathrm{BiS}_{3}\right)$ as a ternary semiconductor nanomaterial possesses broad and strong NIR-II absorption, and thus can be used for NIR-II PTT. Li et al. developed $\mathrm{Cu}_{3} \mathrm{BiS}_{3}$ nanorods via a feasible organic synthesis route, employing oleylamine as a stabilizing agent (Fig. 2f). PEGylated $\mathrm{Cu}_{3} \mathrm{BiS}_{3}$, with NIR-II window absorption and photothermal conversion efficiency of $40.7 \%$, were used as PAI and computer tomography (CT) imaging agents and for imagingguided NIR-II window PTT. ${ }^{51}$ Yang et al. prepared copper manganese sulfide $\left(\mathrm{Cu}_{2} \mathrm{MnS}_{2}\right)$ nanoplates with negligible cytotoxicity, excellent photostability, outstanding photothermal conversion efficiency, and strong absorbance in both the NIR-I and NIR-II windows via a facile one-pot solvothermal method (Fig. 2g). Furthermore, $\mathrm{Cu}_{2} \mathrm{MnS}_{2}$ nanoplates were used as nanoagents for imaging-guided NIR-II window PTT. ${ }^{52}$ Carbonbased nanomaterials as a type of PAI and PT nanoagent have attracted increasing attention due to their wide-band absorption in both the NIR-I and NIR-II windows and their simple functionalization. For example, Wu et al. developed mesoporous carbon nanospheres (Meso-CNs, Fig. $2 \mathrm{~h}$ ) with a wide band and strong absorption across $300-1400 \mathrm{~nm}$ using a silicaassisted synthetic route, and further investigated their potential as a versatile platform for PAI and imaging-guided chemophotothermal therapy.$^{53}$ Silicon (Si), as a type of semiconductor material with LSPR, has attracted much attention for biomedical applications due to its unique optical and electronic properties, abundance, good biodegradability, and biocompatibility. For example, Li et al. designed non-stoichiometric hollow silicon

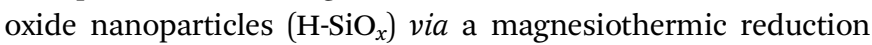
strategy (Fig. 2i). $\mathrm{H}_{-} \mathrm{SiO}_{x}$ exhibited a broadband absorption across $200-1100 \mathrm{~nm}$ and a photothermal conversion efficiency of $48.6 \%$ in the NIR-II region; therefore, it can be used as a nanoagent for PA imaging-guided NIR-II PTT. ${ }^{54}$

\section{Organic materials}

Organic molecular PAI contrast agents and PTT agents have received much attention in recent years, especially conjugated polymer (CP) NPs and semiconducting polymer nanoparticles
(SPN) that have a donor-acceptor (D-A) structure. ${ }^{55-61}$ Due to the strong electron-withdrawing abilities of both CP and SPN, their band gap can be further lowered, resulting in an absorption peak in the NIR-II window. Both CP and SPN have the merits of excellent photostability, good biocompatibility, outstanding imaging contrast, and high extinction coefficient with promising potential for PTT. For example, Pu et al. developed the first wide-band absorbing NIR-II PA organic imaging agent (SPN-II) for direct comparison between the NIR-I vs. NIR-II PA imaging effects and reported that NIR-II PAI offers the advantages of weaker background signals, higher SNR, and better imaging depth (Fig. 3a). ${ }^{62}$ Recently, Pu et al. designed a series of SPN-based metabolizable NIR-II nanoagents with high-performance in vivo for NIR-II window PAI of both deep brain vasculature and subcutaneous tumor at a low dosage (Fig. 3b). ${ }^{63}$ In addition, Mei et al. developed thienoisoindigo (TII)-based SPN (TSPNs) with enhanced absorption across $1000-1350 \mathrm{~nm}$ as a contrast agent for centimeter-deep NIR-II PA imaging (Fig. $3 \mathrm{c}$ and d). ${ }^{15}$ Parmanik et al. designed novel SPNs with absorption in the NIR-II window consisting of ultranarrow bandgap poly (thienoisoindigo-altdiketopyrrolepyrrole) (PIGD) with high structural planarity via the nanoprecipitation method. PIGD as a contrast agent possesses outstanding PA imaging capabilities, and thus can be used for deep-tissue contrast-enhancement in in vivo PAI in the NIR-II window (Fig. 3e). Organic semiconducting polymer nanoparticles (OSPNs) synthesized from semiconducting polymers (SP) are both prospective contrast agents and PTT agents with admirable optical properties for imaging-guided therapy. ${ }^{64}$ Liu et al. designed NIR-II window D-A CP (P1) with excellent biocompatibility, good PA stability, and good imaging ability in both the NIR-I and NIR-II windows via D-A selection (Fig. 3f). ${ }^{65}$ This was the first time that organic molecules were prepared for high-performance NIR-II PAI of orthotopic brain tumors. Subsequently, Liu et al. developed CP NPs with NIR-II absorption via the nanoprecipitation method and subsequent chemical modification with cRGD (an active targeting ligand) for targeted PAI and imaging-guided PTT of brain tumors, penetrating the skull and scalp (Fig. 3g). ${ }^{66}$ Bian et al. developed positively charged OSPNs (OSPNs ${ }^{+}$) consisting of a hydrophobic SP (Fig. 3h) core, an anionic interlayer, and a cationic shell. ${ }^{67}$ The SP core with the strong electron-withdrawing ability of the thiadiazoloquinoxaline unit served as the PA signal generator upon NIR-II and NIR-I light excitation and was prepared via a Pd-catalyzed Stille coupling reaction. Subsequently, water soluble nanoparticles $\left(\mathrm{OSPNs}^{-}\right.$) with a large negative surface charge were prepared by encapsulating amphiphilic poly(styrene maleic anhydride) (PSMA) in the SP core via nanoprecipitation. Poly(L-lysine) (PLL) was strongly attracted onto the OSPNs ${ }^{-}$surface through electrostatic interactions to obtain cationic nanoparticles $\left(\mathrm{OSPNs}^{+}\right)$. The NIR-II window absorptive $\mathrm{OSPN}^{+}$nanoprobe was employed for PA imaging and tracking of stem cells (Fig. 3i).

\section{Other nanocomposites}

In addition to the aforementioned nanoagents, researchers have also developed numerous other nanomaterial-based NIR-II window PA imaging contrast agents and PTT agents, such as 
(a)

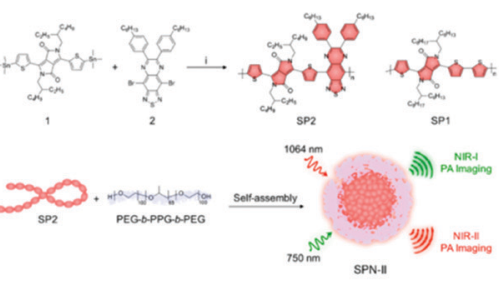

(b)

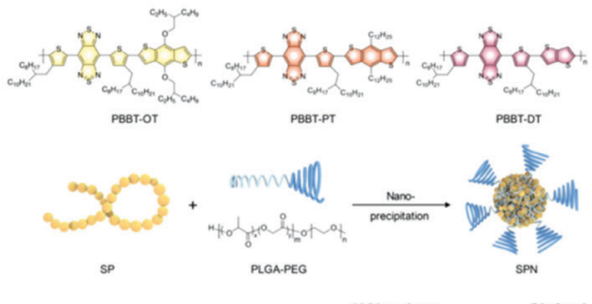

(c)

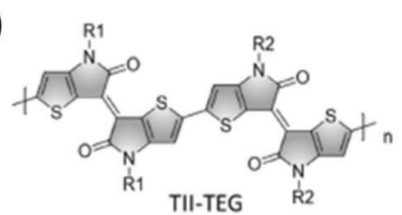

(d)

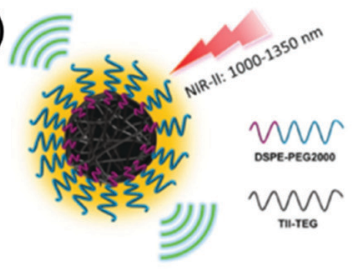

(e)
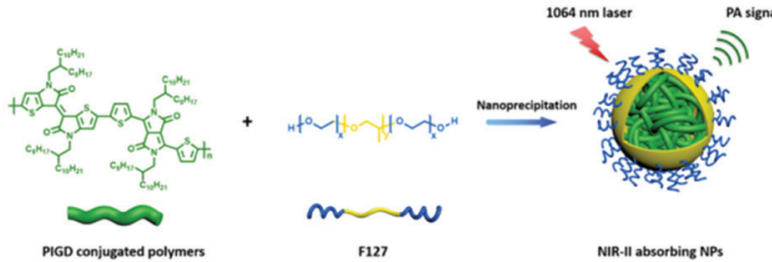

F12

(f)

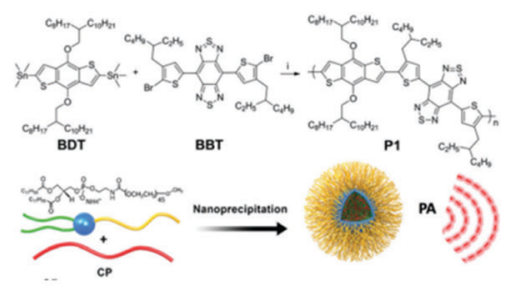

(h)

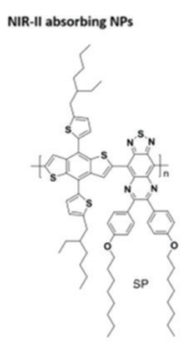

(g)

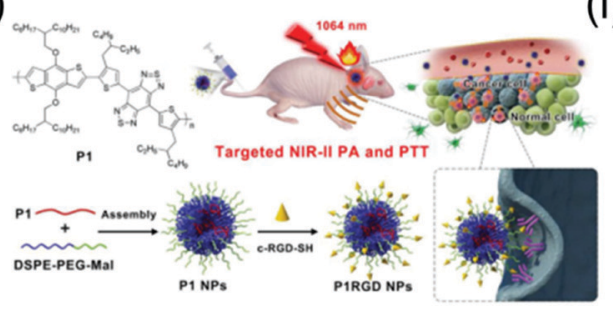

(i)

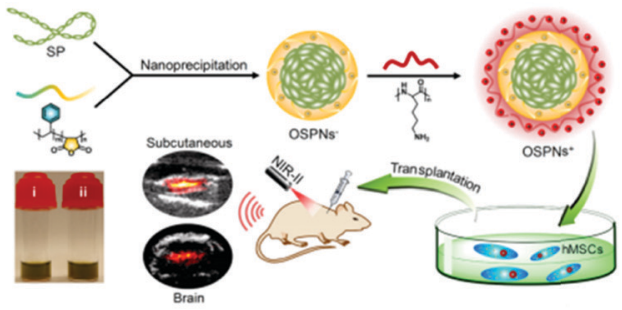

Fig. 3 (a) Chemical structure of SP2 and SP1, and illustration of the fabrication of SPN-II. (b) Chemical structures of PBBT-OT, PBBT-PT, and PBBT-DT, as well as the preparation route of NIR-II PA SPNs. (c) Chemical structure of TII-TEG. (d) Proposed mechanism of TSPN prepared by nanoprecipitation. (e) Proposed mechanism of NIR-II absorbing NPs. (f) Preparation of P1 and its self-assembly via nanoprecipitation. (g) Schematic illustration of the fabrication of P1RGD nanoparticles (NPs) and their application in brain tumor treatment. (h) Chemical structure of SP. (i) Schematic illustration of the synthetic route of OSPNs ${ }^{+}$ and their in vivo application. (f) was reproduced from ref. 65 with permission from the Royal Society Chemistry, Copyright 2017, (g) was reproduced from ref. 66 with permission from Wiley- $\mathrm{VCH}$, Copyright 2018, (a) was reproduced from ref. 62 with permission from the American Chemical Society, Copyright 2017, (b) was reproduced from ref. 63 with permission from Wiley-VCH, Copyright 2019, (c and d) were reproduced from ref. 15 with permission from Wiley-VCH, Copyright 2017, (e) was reproduced from ref. 68 with permission from the Society of Photo-Optical Instrumentation Engineers, Copyright 2019 and (h and i) were reproduced from ref. 67 with permission from the American Chemical Society, Copyright 2018.

perfluorocarbon nanodroplets, ${ }^{24}$ phosphorus phthalocyanine, ${ }^{25}$ and two-dimensional (2D) materials. ${ }^{69}$ MXenes, consisting of transition metal carbides and either carbonitrides or nitride, have received much attention for biomedical applications due to their excellent biocompatibility, natural hydrophilicity, enhanced absorption in the NIR window, and ready functionalization. For example, Zhang et al. developed 2D vanadium carbide quantum dots $\left(\mathrm{V}_{2} \mathrm{C}\right.$ QDs, Fig. 4a) with enhanced photothermal effect and PA imaging contrast in the NIR-II window as well as MR imaging ability via a liquid exfoliation and hydrothermal strategy. ${ }^{69}$ A multifunctional theranostic platform ( $\mathrm{V}_{2} \mathrm{C}$-TAT@Ex-RGD) with long circulation time, excellent biocompatibility, and endosomal escape ability was constructed, through $\mathrm{V}_{2} \mathrm{C}$ QDs modified with cell nucleus-target TAT peptides, which was packaged into endogenous exosomes (Ex) with RGD modification (Fig. 4b). The nanoagents could target cells and enter the nucleus to achieve low temperature PTT with high cancer destruction efficiency. $\mathrm{TiO}_{2}$ with a wide bandgap of $3.2 \mathrm{eV}$ is a semiconductor nanomaterial with good biomedical application prospects due to its excellent biocompatibility. However, semiconductors with such a wide bandgap can only absorb ultraviolet and visible light rather than NIR light, and therefore it is difficult to use $\mathrm{TiO}_{2}$ as an NIRresponsive nanoagent. However, its absorption band can be effectively adjusted to the NIR region through the Nb-doping method. ${ }^{70}$ An Nb-doped $\mathrm{TiO}_{2}$ nanoagent could convert light energy into heat under the illumination of a $1064 \mathrm{~nm}$ laser; thus, PTT can be realized. $\mathrm{WO}_{3-x}$ nanostructures with strong NIR absorption rooted in the LSPR of free electrons have been used as PTT agents; ${ }^{71}$ however, their PTT in the NIR-II window has rarely been developed due to insufficient photothermal conversion efficiency. The NIR absorption and photothermal conversion efficiencies can be enhanced by doping heterogeneous atoms to 
(a)

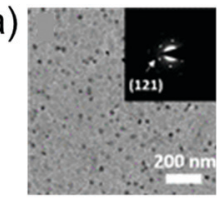

(c)

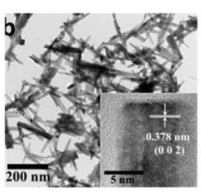

(b)

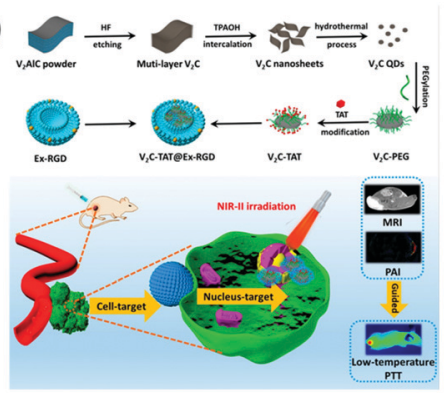

(d)

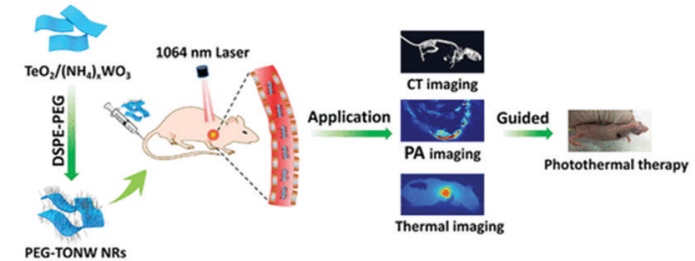

(e)

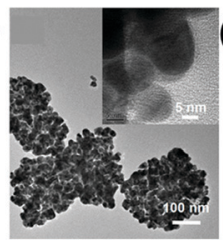

(f)

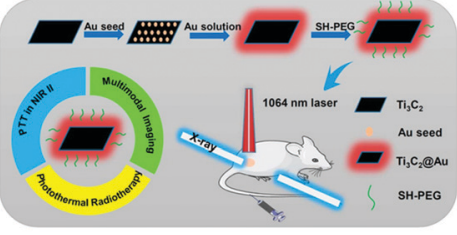

Fig. 4 (a) TEM images of $V_{2} C Q D s$, (b) synthetic route of the $V_{2} C$-TATa Ex-RGD, and multimodal imaging-guided NIR-II photothermal therapy, (c) TEM image of TONW NRs, (d) proposed mechanism of the applications of PEG-TONW NRs, (e) TEM image of $\mathrm{Ti}_{3} \mathrm{C}_{2} \mathrm{QAu}$ nanocomposites, and ( $f$ ) schematic illustration of the synthetic route and dual-modal imaging-guided cancer therapy. ( $a$ and $b$ ) were reproduced from ref. 69 with permission from the American Chemical Society, Copyright 2019, (c and d) were reproduced from ref. 70 with permission from the American Chemical Society, Copyright 2019 and (e and f) were reproduced from ref. 73 with permission from the American Chemical Society, Copyright 2019.

construct $\mathrm{M}_{x} \mathrm{WO}_{3}$. For example, Zhang et al. designed 2D ultrathin tellurium oxide/ammonium tungsten bronze nanoribbons (TONW NRs, Fig. 4c) with enhanced PA imaging ability and advanced photothermal conversion efficiency in the NIR-II window. They used a liquid-liquid interface-mediated strategy for CT and PA imaging and imaging-guided NIR-II PTT cancer treatment (Fig. 4d). ${ }^{72}$ Cheng et al. developed multifunctional 2D nanocomposites $\mathrm{Ti}_{2} \mathrm{C}_{3}$ @Au (Fig. 4e) with a core-shell structure via a seed-growth strategy. ${ }^{73} \mathrm{Ti}_{2} \mathrm{C}_{3} @ A u$ nanocomposites have the merits of improved stability and biocompatibility, enhanced optical absorbance ability in both the NIR-I and NIR-II windows, as well as an outstanding optical performance and advanced X-ray attenuation capability. Therefore, they can be used as nanoagents for CT and PA dual-modal imagingguided NIR-II PTT and photothermal radiotherapy (Fig. 4f).

Different types of PA imaging nanoagents possess different advantages and disadvantages. Inorganic materials such as metallic nanomaterials have the advantages of facilely adjustable biochemical and physiochemical properties and excellent biocompatibility, and disadvantages of poor biodegradability. 2D nanomaterials such as carbon-based or transition-metal chalcogenides/MXene-based nanomaterials have the merits of good photothermal stability, high drug-loading content and drawbacks of needing surface modification and poor biodegradability. Organic nanomaterials have the advantages of excellent optical properties and biocompatibility, low toxicity, high photostability, fast clearance, and controllable size, and disadvantages of short in vivo circulation time, poor aqueous solubility and unknown biodegradation behaviour.

\section{Photoacoustic imaging in the second near-infrared window}

\section{Comparison of photoacoustic imaging effects between first and second near-infrared window}

The NIR-II window possesses intrinsic merits of deeper tissue penetration and higher MPE due to decreased tissue scattering and decreased energy of long wavelength photons, respectively. Compared to the NIR-I window, NIR-II light has been reported to offer excellent spatial resolution and enhanced SBR for PA imaging. ${ }^{74,75}$ For example, Yuan et al. designed CuS NPs with a broadband absorption ranging from 600 to $1350 \mathrm{~nm}$ (Fig. 5a), which acted as excellent contrast agents for multispectral PAI. ${ }^{76}$ In a phantom experiment, an agar gel phantom containing $\mathrm{CuS}$ NPs was buried in chicken breast tissues at different depths, and the penetration depths of the NIR-I and NIR-II windows by PA imaging were measured with a laser density of $5 \mathrm{~mJ} \mathrm{~cm}^{-2}$. Yuan et al. reported that the PA signal intensity of CuS decreased with an increase in depth in both the NIR-II and NIR-I regions. For larger SNR, stronger imaging contrast and higher penetration depths upon illumination with a $1050 \mathrm{~nm}$ laser were found compared to that of a $700 \mathrm{~nm}$ laser. In addition, when CuS NPs were intravenously injected into tumor-bearing mice in an in vivo experiment, the PA signal gradually increased in both the NIR-I and NIR-II windows. Especially in the NIR-II window, the signal intensity at $5 \mathrm{~h}$ post-injection was about two times of that at pre-injection (Fig. 5b). Furthermore, the SNR in the NIR-II window was higher than that in the NIR-I window (Fig. 5c). Pu et al. developed the first SPN-based PA contrast agent with broadband absorption in both the NIR-I and NIR-II windows, ${ }^{62}$ encapsulating SP in an amphiphilic copolymer (PEG- $b$-PPG- $b$-PEG) (Fig. 3c) to form SPN via a self-assembly approach. Compared to SP1, with a maximum absorption at $744 \mathrm{~nm}, \mathrm{SP} 2$ with thiadiazoloquinoxaline showed more powerful electron-withdrawing ability; thus, further reducing the band gap and yielding the maximum absorption peak at $1253 \mathrm{~nm}$ in the NIR-II window (Fig. 5d). Thus, SP2 can function as a PA contrast agent for biomedical imaging in both the NIR-I and NIR-II regions. Due to the higher MPE of a $1064 \mathrm{~nm}$ laser compared to a $750 \mathrm{~nm}$ laser, the imaging depth was deeper when the former was used. Up to $3 \mathrm{~cm}$ of tissue imaging depth at both the NIR-I and NIR-II windows could be achieved with a laser of $5.5 \mathrm{~mJ} \mathrm{~cm}^{-2}$, while an imaging depth of 4 and $5 \mathrm{~cm}$ at NIR-II window was also achieved when using a laser power density of $20 \mathrm{~mJ} \mathrm{~cm}^{-2}$ (Fig. 5e). In addition, the SBR for NIR-II imaging was higher than that for NIR-I imaging for all depths (Fig. 4f). An in vivo experiment of brain imaging in living 

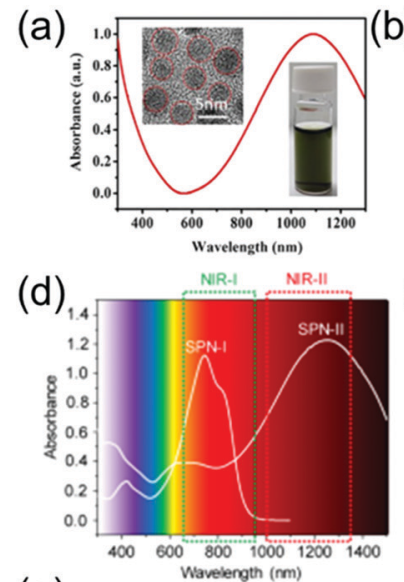

(g)

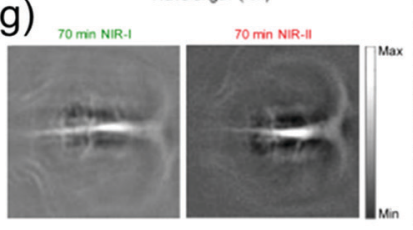

(b)

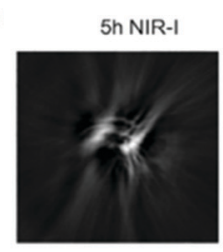

(e)

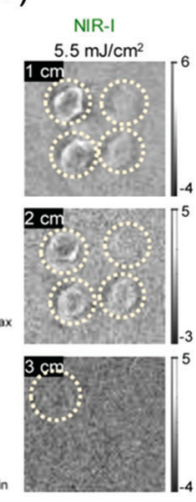

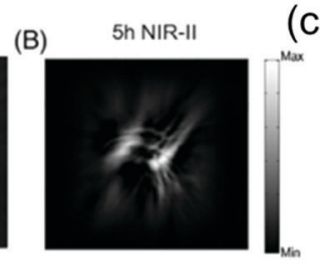
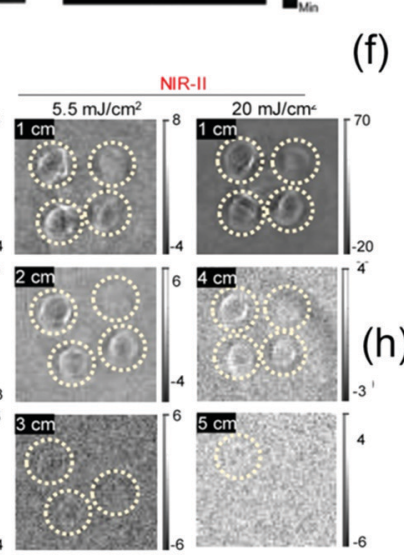

(f) (c)
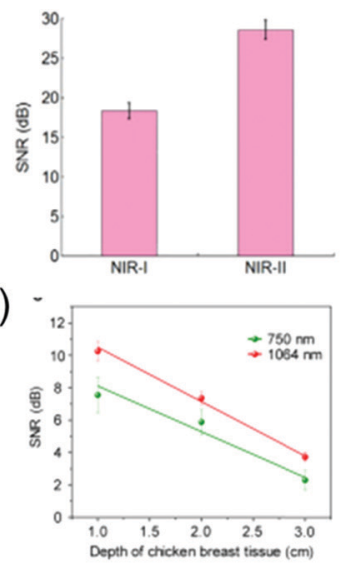

(h)

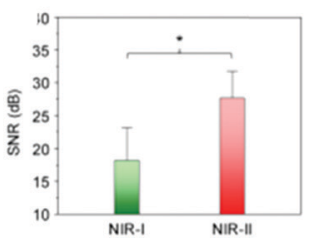

Fig. 5 (a) Absorption spectra of CuS nanoparticles, (b) in vivo PA tumor imaging, (c) SNR of tumor images in the NIR-I and NIR-II windows, (d) absorption spectra of SPN-I and SPN-II, (e) PA images of an agar gel phantom containing SPN-II solutions at different depths, (f) SNR at 1064 or 750 nm as a function of chicken breast tissue depth, (g) PA images of brain vasculature at different wavelengths and (h) SNR of the NIR-I and NIR-II windows. (a-c) were reproduced from ref. 76 with permission from Wiley- $\mathrm{VCH}$, Copyright 2018 and (d-h) were reproduced from ref. 62 with permission from the American Chemical Society, Copyright 2017.

rats exhibited a 1.5-fold higher SNR for the NIR-II window than that of NIR-I PA imaging (Fig. $5 \mathrm{~g}$ and $\mathrm{h}$ ).

\section{Subcutaneous tumor imaging}

Subcutaneous tumor imaging is one of the most commonly used experimental methods for biomedical application. For example, Mei et al. developed thienoisoindigo (TII)-based SPNs (TSNPs) with a broadband absorption from 600-1800 nm (Fig. 6a) and enhanced PA signals in the NIR-II window. ${ }^{15}$ The TSNPs consisted of TII-based SP-attached triethylene glycol chains (Fig. 3e and f). The as-prepared TSNPs exhibited outstanding PA imaging ability in both the NIR-I and NIR-II windows and significantly minimized the background signals of endogenous chromophores in the NIR-II window (Fig. 6b and c). In response to illumination with a $1064 \mathrm{~nm}$ laser and at contrast agent concentrations as low as $40 \mu \mathrm{g} \mathrm{mL}^{-1}$, the imaging depth of chicken-breast tissue with a TSNP-containing tube exceeded $5 \mathrm{~cm}$. Therefore, TSNP, with a PA imaging ability of several centimeter depth, can be used as a suitable NIR-II contrast agent. Compared to the NIR-I window, the PA intensity of the biological tissue itself in the NIR-II region is considerably lower. For skin tissue without injection of TSNP-containing contrast agent, only a negligible PA signal was recorded at $1100 \mathrm{~nm}$ and $1300 \mathrm{~nm}$ (NIR-II window), while a higher PA signal intensity was observed at $800 \mathrm{~nm}$ (NIR-I window). Additionally, the PA signal was enhanced approximately 7.3- and 10.7-fold at $1100 \mathrm{~nm}$ and $1300 \mathrm{~nm}$, respectively, following injection of $50 \mu \mathrm{L}$ of $40 \mu \mathrm{g} \mathrm{mL}{ }^{-1}$ TSNP solution, compared to the PA signal of skin tissue without contrast agent. In vivo PA imaging of tumor-bearing mice was performed. Without TSPN injection, the strong PA signal from the tumor tissue appeared only at $800 \mathrm{~nm}$ and $1000 \mathrm{~nm}$ due to the abundance of blood vessels around the tumor. The PA signal was improved by about 7.0- and 13.3-fold at $1100 \mathrm{~nm}$ and $1300 \mathrm{~nm}$, respectively, following intratumoral injection of $50 \mu \mathrm{L}$ of $40 \mu \mathrm{g} \mathrm{mL}{ }^{-1}$ TSNP solution compared to the PA signal of the tumor without contrast agent (Fig. 6d). NIR-II PA contrast agents and photothermal agents for application in the tumor microenvironment have rarely been reported. Hypoxia is a typical characteristic of tumors, which threatens physiological function; thus, imaging of hypoxia can be employed as a predictor of cancer and can guide the therapeutic regimen. Cai et al. designed a hypoxia-responsive and nitroreductase enzymeresponsive single molecule nanoagent for NIR-II window PA tumor imaging with high contrast and hypoxia-triggered PTT. ${ }^{77}$ They achieved higher tissue penetration depth and increased accuracy of the imaging of the boundary and position of tumors. Importantly, the hypoxia-triggered PTT of the nanoagent showed excellent phototherapeutic efficacy with rapid temperature increase and led to the ablation of tumors without recurrence. Through abnormal energy metabolism and selfregulation of specific proteins, tumor cells form and maintain an extracellular acidic microenvironment that is unsuitable for the survival of normal cells, but ensures the occurrence, proliferation, invasion, and metastasis of tumor cells. The acidic environment created by the tumor is of great use for the growth of the tumor. It can change the function of macrophages, promote tumor growth, inhibit the activity of killer $\mathrm{T}$ cells, and create an environment that is free from immune system agents, all of which benefit cancer cells. ${ }^{78}$ Therefore, it is very meaningful to design PA probes that have a specific response to the acidic 
(a)

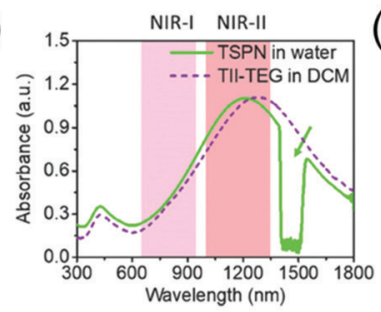

(b)

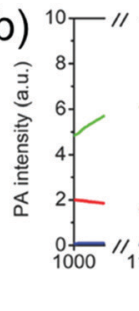

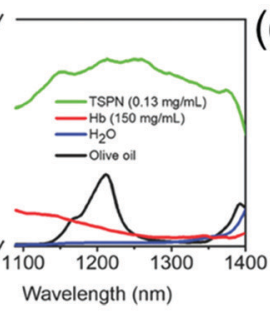
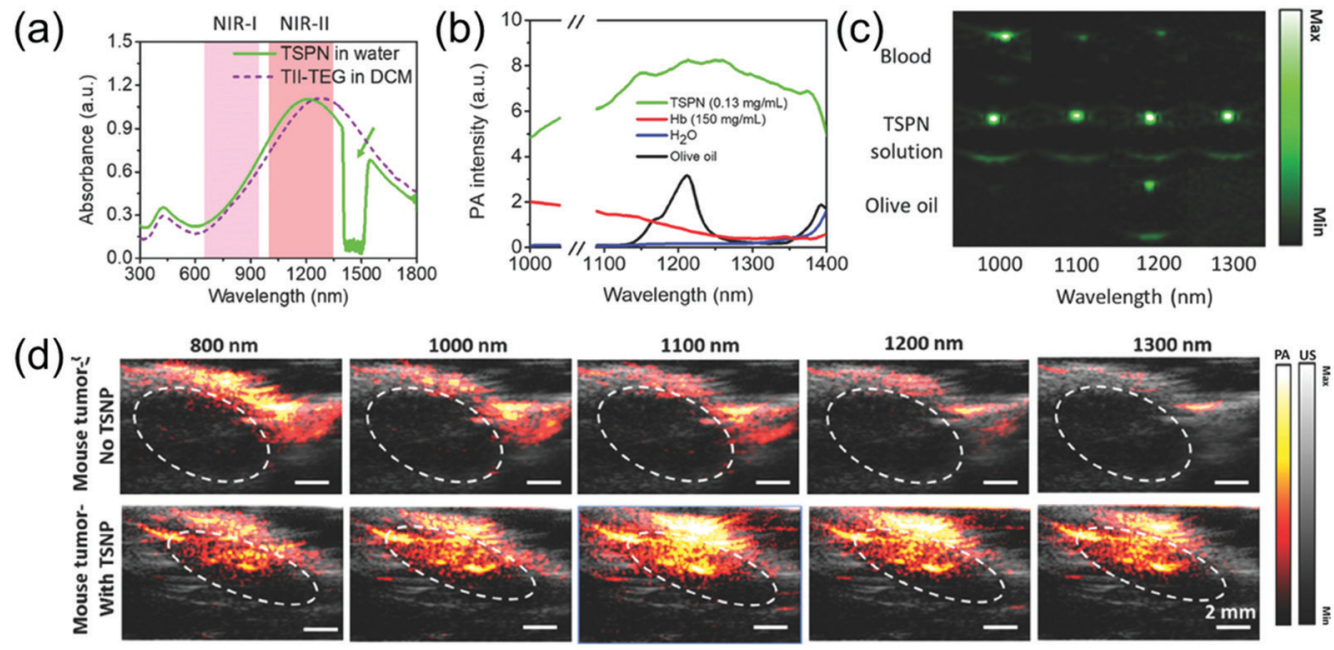

(e)
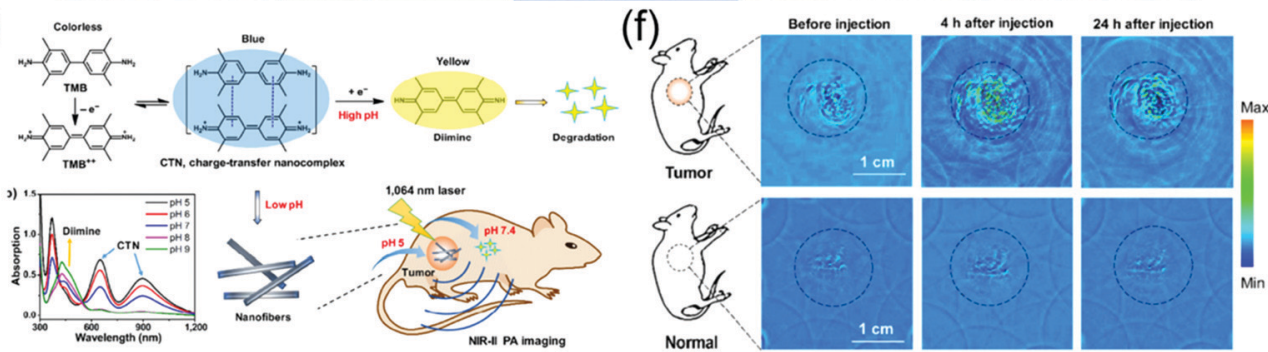

Fig. 6 (a) UV-vis-NIR absorption spectrum of TSPN, (b) spectra of water, hemoglobin ( $\mathrm{Hb}$ ), olive oil (lipid), and TSPNs, (c) PA images of blood, TSPNs, and olive oil in plastic tubes at different laser wavelengths, (d) in vivo PA/US tumor imaging of TSPN, (e) proposed mechanism of CTN preparation, degradation, and in vivo PA imaging and (f) in vivo PA tumor images in the NIR-II window. (a-d) were reproduced from ref. 15 with permission from WileyVCH, Copyright 2017 and (e and f) were reproduced from ref. 79 with permission from Springer Nature, Copyright 2018.

environment of tumors. For example, Xing et al. developed a novel $\mathrm{pH}$-sensitive NIR-II window organic charge-transfer nanocomplex (CTN) PA probe that is biodegradable under physiological conditions through a self-assembly method using $3,3^{\prime}, 5,5^{\prime}$-tetramethylbenzidine (TMB) and its dication structure $\left(\mathrm{TMB}^{++}\right)$. The CTN probe responded specifically to a variation in acidity and permitted the sensitive and noninvasive visualization of the tumorbearing mice acidic environment with a high SNR (Fig. 6e). ${ }^{79}$ In vivo PA imaging of tumor-bearing mice with intratumoral injection of $200 \mu \mathrm{L}$ of $1 \mathrm{mg} \mathrm{mL}{ }^{-1}$ CNT was compared to in vivo PA imaging of control mice with subcutaneous injection of the same dosage CNT. Both the control and tumor-bearing mice only showed background signals at pre-injection due to tissue absorption in the NIR-II window. The PA signal in the tumor increased significantly with time due to the response of the CTN probe in the acidic tumor environment. After $4 \mathrm{~h}$, a very distinct difference in PA signal was found between normal tissue and tumor. At $24 \mathrm{~h}$ post-injection, the tumor-bearing mice still exhibited marked PA signals; however, the control mice showed no PA signal because CNT could be degraded at physiological pH levels (Fig. 6f).

\section{Deep brain vasculature imaging}

The brain is the most important organ of living organisms, and if disease occurs here, the consequences will be very severe. Brain vascular imaging technologies such as CT and MRI have been developed in simple and non-invasive methods for the diagnosis of various intracranial vascular diseases. These methods offer the advantages of simple examination method, no special preparation required, short scanning time, and high imaging resolution. Furthermore, lesions can be suitably displayed via many types of image post-processing technologies. However, research on the PA imaging of brain vasculature is still being developed. Pramanik et al. developed PIGD with a wide-band absorption band ranging from $600 \mathrm{~nm}$ to $1200 \mathrm{~nm}$ (Fig. 7a) as an NIR-II contrast agent for the PA imaging of rat brain vasculature in vivo. ${ }^{68}$ By burying the PIGD probe in chicken-breast tissue, a PA imaging depth of approximately $5 \mathrm{~cm}$ could be achieved upon irradiation with a $1064 \mathrm{~nm}$ laser. Inspired by this, an in vivo brain vasculature imaging experiment was conducted, where the rat brain vascular was imaged via an PA imaging system in the NIR-II window following injection of $0.25 \mathrm{~mL}$ of $2 \mathrm{mg} \mathrm{mL} \mathrm{m}^{-1}$ PIGD probe. The imaging contrast of the brain vascular was poor because the blood at $1064 \mathrm{~nm}$ only possessed low absorption. However, the PA signal and SNR gradually increased over time after injection, reaching a peak at $70 \mathrm{~min}$ (Fig. $7 \mathrm{~b}$ and $\mathrm{c}$ ). As the main organ of the central nervous system, the brain plays an important role in the study of neuron-related pathology. However, due to the strong scattering caused by the skull, the imaging of the brain with optical contrast agents remains challenging. However, the light scattering of the skull in the NIR-II window is greatly reduced; therefore, NIR-II PAI provides a promising solution to overcome 
(a)

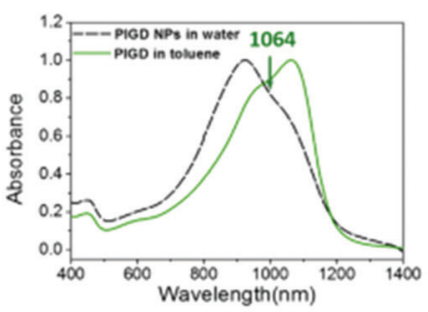

(c)

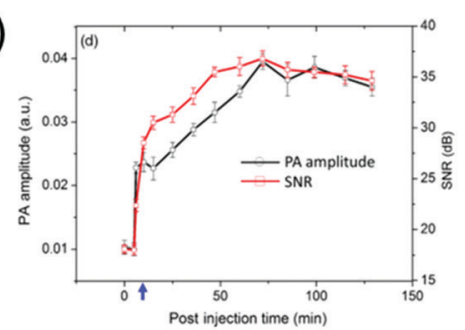

(e)

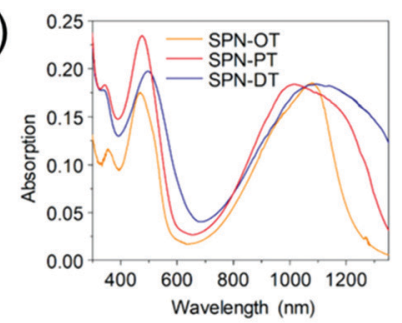

(b)

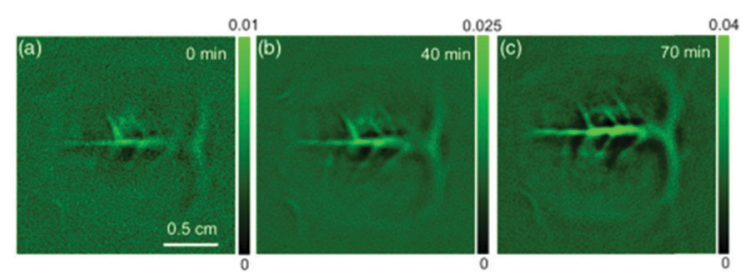

(d)

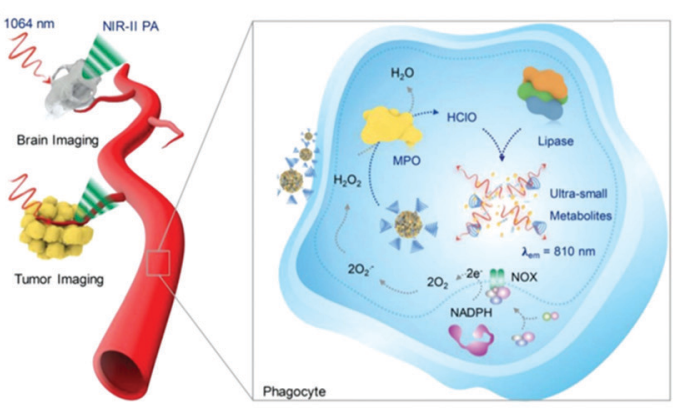

(h)
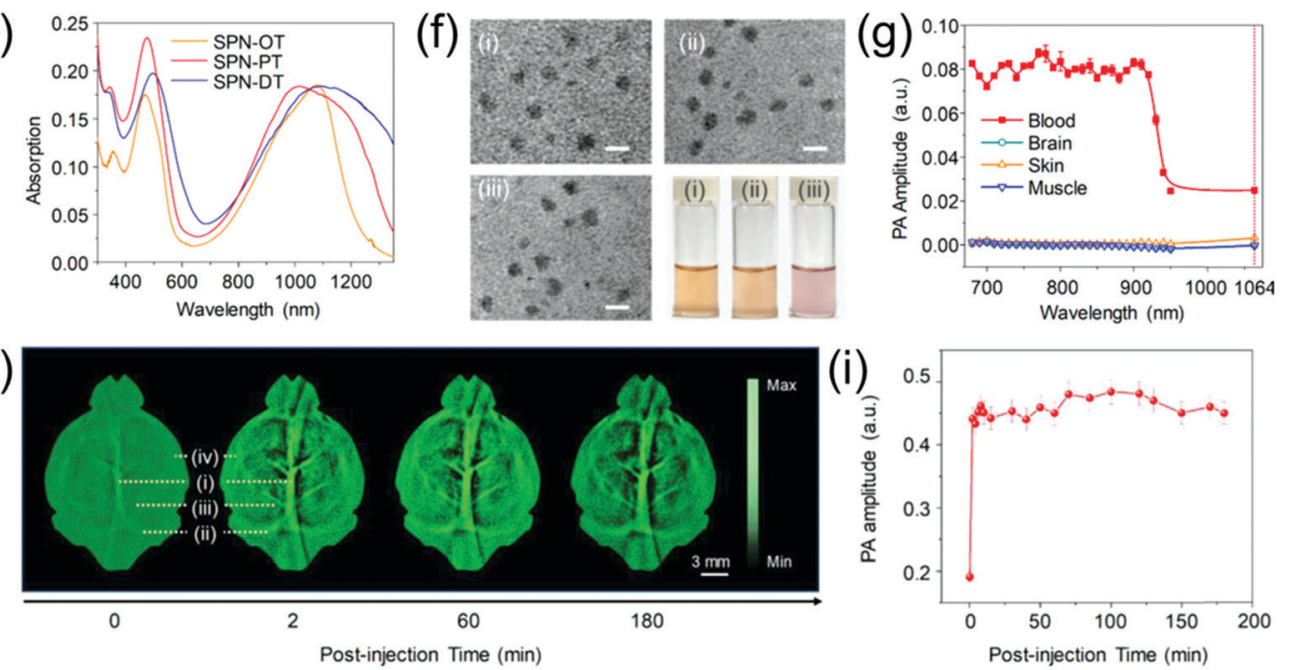

Fig. 7 (a) Absorption spectrum of PIGD, (b) in vivo PA rat brain vasculature imaging, (c) PA spectra and SNR as a function of post-injection time, (d) proposed mechanism of NIR-II PA imaging of tumor and brain as well as in vivo biodegradation of SPNs, (e) normalized absorption spectra of SPN-PT, SPN-OT, and SPN-DT, (f) TEM images and photographs of a series of SPNs, (g) PA amplitude of muscle, skin, brain cortex, and blood, (h) PA images of brain vasculature at $1064 \mathrm{~nm}$ and (i) quantification of PA signal of blood vessels in panel (h). (a-c) were reproduced from ref. 68 with permission from the Society of Photo-Optical Instrumentation Engineers, Copyright 2018; (d-i) were reproduced from ref. 63 with permission from Wiley-VCH, Copyright 2019.

this problem. For example, $\mathrm{Pu}$ et al. designed a series of metabolizable SPN-based NIR-II PA probes for the imaging of both deep brain vasculature and subcutaneous tumors (Fig. 7d). ${ }^{63}$ The SPN-based nanoprobes (named SPN-PT, SPN-OT, and SPN-DT) with a spherical morphology (Fig. 7f) had a wide-band absorption ranging from $700 \mathrm{~nm}$ to $1350 \mathrm{~nm}$ (Fig. 7e). Among them, the photothermal conversion efficiencies of SPN-PT was highest (53\%). Therefore, SPN-PT as a probe was used in subsequent in vitro and in vivo experiments. To emphasize the potential merits of NIR-II PAI over NIR-I PAI, the PA amplitude of the brain cortex, fresh blood, muscles, and skin was tested (Fig. 7g). The PA amplitude of biological tissues, including the above-mentioned four categories in the NIR-II region, showed a marked decrease compared to that of the NIR-I window. The PA images of subcutaneous tumors at different time points in the NIR-II window after intravenous injection of $200 \mu \mathrm{L}$ of $250 \mu \mathrm{g} \mathrm{mL} \mathrm{m}^{-1} \mathrm{SPN}-\mathrm{PT}$ probe showed that the PA signal intensities in the tumor areas were gradually enhanced over time, reaching the maximum at $8 \mathrm{~h}$ post-injection. Subsequently, NIR-II PA images of deep brain vasculature were obtained, and the PA signal intensities of the brain vasculature immediately enhanced, resulting in an excellent contrast between background parenchyma and brain vasculature. The superior sagittal sinus (i in Fig. 7h), transverse sinus (ii in Fig. 7h), vascular branches (iii in Fig. 7h), and middle cerebral arteries (iv in Fig. 7h) could be clearly observed at 2 min post-injection. Furthermore, the PA signal still remained visible at $3 \mathrm{~h}$ post-injection due to the long blood circulation of SPN-PT (Fig. 7e).

\section{Orthotopic tumor imaging}

The NIR-II PA imaging of deep orthotopic tumors has gradually attracted attention. For example, Zhang et al. designed ultrasmall tumor-targeted protein-CuS nanoagents (CuS@BSA-RGD) with low cytotoxicity, efficient cellular uptake, and negligible hemolysis, which was the first time that PA imaging of orthotopic 
(a)

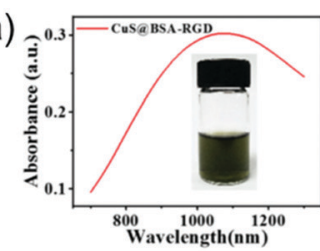

(b)

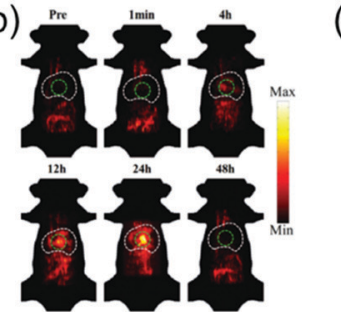

(h)

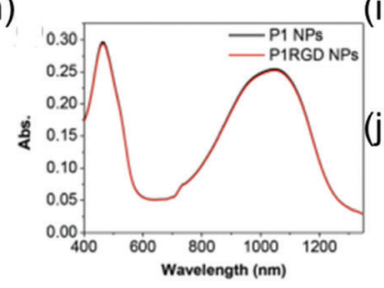

(c)

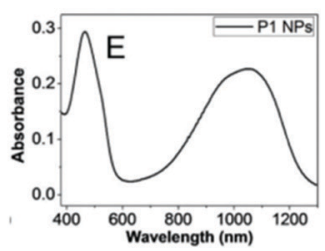

(f)

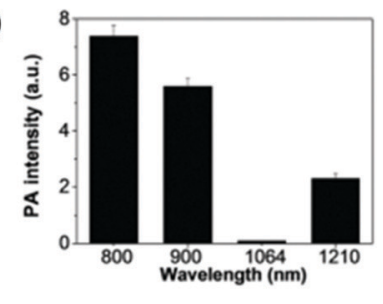

(d)

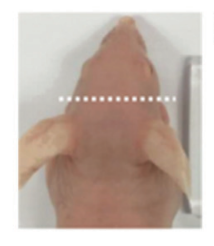

(e)

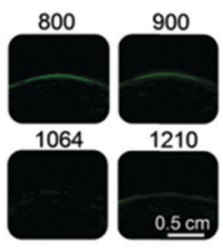

(g)

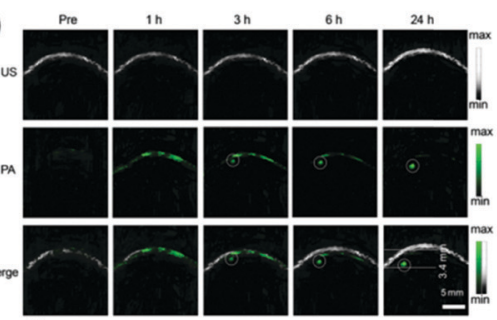

(i)
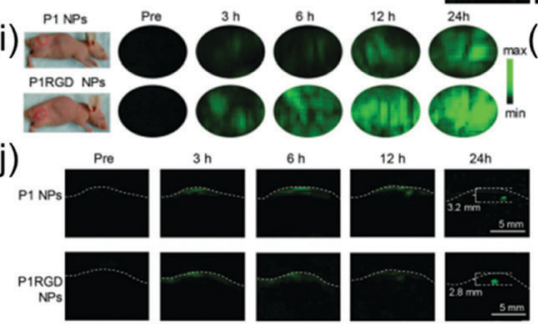

(k)

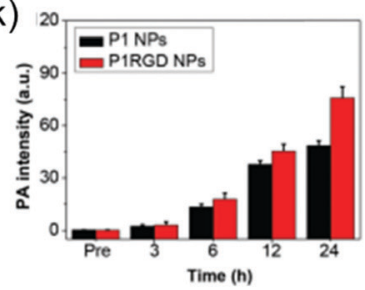

Fig. 8 (a) UV-vis-NIR absorption of CuS@BSA-RGD NPs, (b) in vivo orthotopic liver cancer photoacoustic imaging of CuS@BSA-RGD NPs, (c) absorption spectra of P1 NPs, (d) photograph of tumor-bearing mice, (e) PA scanning of brain of tumor-bearing mice at different excitation wavelengths, (f) PA background signal intensity of the mouse brain at different wavelengths, (g) PA images of the brain tumor at both pre- and post-injection, (h) absorption spectrum of P1 NPs and P1RGD NPs, (i) in vivo PA imaging of the subcutaneous U87 tumor model at different time points, (j) PA imaging of a mouse brain tumor through the scalp and skull and (k) quantitative results of brain tumor PA intensity. (a and b) were reproduced from ref. 50 with permission from the Royal Society of Chemistry, copyright 2019, (c-g) were reproduced from ref. 65 with permission from the Royal Society of Chemistry, Copyright 2017 and $(\mathrm{h}-\mathrm{k})$ were reproduced from ref. 66 with permission from Wiley-VCH, Copyright 2018.

hepatocellular carcinoma was used and achieved excellent SNR. ${ }^{50}$ The CuS@BSA-RGD NPs with good dispersibility and stability had a wide-band absorption ranging from $800 \mathrm{~nm}$ to $1350 \mathrm{~nm}$ (Fig. 8a). An in vitro experiment showed that the CuS@BSA-RGD NPs exhibited lower background noise and higher SNR due to negligible tissue absorption and scattering in the NIR-II window. PA images of orthotopic hepatocellular carcinoma at different time points in the NIR-II window after intravenous injection of $100 \mu \mathrm{L}$ of $1 \mathrm{mg} \mathrm{mL} \mathrm{m}^{-1}$ CuS@BSA-RGD probe showed a low PA amplitude in the abdominal and thorax at pre-injection because blood vessels and intestine surround the liver. After injection, the PA signal of the orthotopic hepatocellular carcinoma gradually enhanced and reached a maximal value at $24 \mathrm{~h}$ post-injection due to target accumulation of CuS@BSA-RGD (Fig. 8b). In addition, Li et al. developed a CuS nanoagent that can be used for PA imaging of deep orthotopic brain tumors. ${ }^{23}$ The CP nanoparticles possess excellent biocompatibility, high imaging contrast, outstanding photostability, and large extinction coefficients. Therefore, they can be used for NIR-II PA imaging of orthotopic brain tumors. For example, Liu et al. developed a NIR-II window D-A CP (P1) with excellent biocompatibility, good PA stability, wide-band absorption, and imaging ability in both the NIR-I and NIR-II windows through D-A selection (Fig. 8c). This was successfully applied to NIR-II PA imaging of an orthotopic brain tumor. ${ }^{65}$ To select the optimal imaging laser wavelength, PA imaging of U87 tumor-bearing mice with different wavelengths was performed (Fig. 8d). The results showed that PA imaging of brain tumors at $1064 \mathrm{~nm}$ lowered the background interference (Fig. 8e and f). Encouraged by the admirable $1064 \mathrm{~nm}$ PA imaging ability of P1 NPs, in vivo NIR-II PA imaging of an orthotopic brain tumor was assessed. Since skin and skull tissue possess ultra-low extinction coefficients at $1064 \mathrm{~nm}$, an extremely low PA background signal was observed. However, no signal of the brain tumor was detected prior to P1 probe injection. An enhanced PA signal surrounding the skin and skull was observed at $1 \mathrm{~h}$ postinjection, suggesting intravascular circulation of the $\mathrm{P} 1$ probe. The PA signal of brain tumor could be clearly observed at $2 \mathrm{~h}$ post-injection and the imaging depth of the brain tumor region rapidly increased over time. A strong PA signal was detected at an imaging depth of up to $3.4 \mathrm{~mm}$ after $24 \mathrm{~h}$ injection, and the PA signal at $24 \mathrm{~h}$ post-injection was improved by about 94 -fold compared to before injection (Fig. 8g). The results successfully illustrated that the P1 probe could pass through the blood brain barrier and accumulate in the brain tumor. Furthermore, Liu et al. designed CP NPs (P1) with wide-band absorption in both the NIR-I and the NIR-II windows (Fig. 8h) via the nanoprecipitation route and subsequent functionalization with cRGD (P1RGD) for targeted PAI and imaging-guided PTT of orthotopic brain tumors. ${ }^{66}$ In the study of subcutaneous tumor models, the PA signal of the P1RGD probe group was approximately 2-fold higher than that of the $\mathrm{P} 1$ probe group $3 \mathrm{~h}$ 
after injection. Due to the active targeting triggered by RGD, the PA signal reached a peak value and the signal/background ratio of P1RGD probe group was about 1.85-fold that of the P1 probe group after $24 \mathrm{~h}$ injection (Fig. 8i). In the study of orthotopic brain tumor models, prior to injection, the PA background signals from the skull, adjacent to the tumor, blood, and tissue around the tumor were not detected, indicating the superiority of the $1064 \mathrm{~nm}$ laser for PA imaging of tumor. Strong PA signals surrounded the skin and skull at $3 \mathrm{~h}$ post-injection, suggesting intravascular circulation of the P1 contrast agent. Consequently, the PA signal reached maximum both in the P1RGD group and P1 group and imaging depths of up to 2.8 and $3.2 \mathrm{~mm}$ were achieved, respectively, at $24 \mathrm{~h}$ post-injection. The signal/background ratio of the P1RGD group was approximately 1.5-fold that of the P1 group due to the active brain-tumor targeting by the c-RGD NPs.

\section{Stem cell imaging}

Human mesenchymal stem cells (hMSCs) have been widely studied in the biomedical field due to their pluripotency. Currently, fluorescence imaging, positron emission tomography, and magnetic resonance imaging are commonly used for stem cell imaging and tracking; however, they have disadvantages. PAI is a living body optical imaging technique with higher imaging depth and spatial resolution. However, the low biological safety of PA contrast agents and strong scattering in the NIR-I imaging limit its application in imaging and tracking of stem cells. PAI in the NIR-II window achieves deeper tissue penetration and higher MPE, which are beneficial for stem cell imaging and tracking. Bian et al. developed a OSPN ${ }^{+}$nanoprobe with a spherical structure (Fig. 9a) and reported two almost identical absorption intensity peaks at 1025 and $916 \mathrm{~nm}$ (Fig. 9b) for PA imaging and tracking of stem cells. $^{67}$ The OSPN ${ }^{+}$nanoprobe had excellent stability and its PA intensity was almost invariable even after irradiation with 24000 laser pulses (Fig. 9c). The $\mathrm{OSPN}^{+}$nanoprobe possesses excellent labeling efficacy due to the interactions between the negatively charged cell membrane and the positively charged nanoprobe. Consequently, the PA intensities of the OSPN ${ }^{+}$-labeled cell pellets was about 3-fold higher than that of the OSPNs${ }^{-}$-labeled cell pellets (Fig. 9c and d). The superiority of PA imaging in the NIR-II window relative to the NIR-I window was tested in a chicken breast tissue model. As shown in Fig. 9f, the OSPNs ${ }^{+}$achieved better imaging capacity and a higher SNR (Fig. 9g) in the NIR-II window compared to the NIR-I window. In vivo studies showed that the NIR-II PAI of OSPN ${ }^{+}$-labeled hMSCs increased by 40.6 and 21.7 times after subcutaneous and brain transplantation, respectively, compared to the unlabeled control group (Fig. $9 \mathrm{~h}$ and i). In addition, PA imaging in the NIR-II window showed a higher SNR than that in the NIR-I region (1.38 times) in deep brain tissue (Fig. 9i), which further corroborates its superiority for the labeling and tracking of stem cells.

\section{Photothermal therapy in the second near-infrared window}

PTT is a potential alternative or adjunct to traditional cancer therapy. PTT is a laser irradiation light absorbing technique that kills cancer cells and has recently entered the clinical trial phase. The factors that affect the photothermal ablation of solid tumors are mainly the absorption of the PTT agent in the NIR region and the penetration depth of the tumor tissue. ${ }^{65,80-84}$ Currently, PTT in the NIR-II window has received increasing

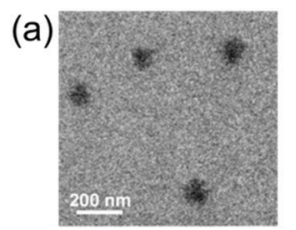

(b)
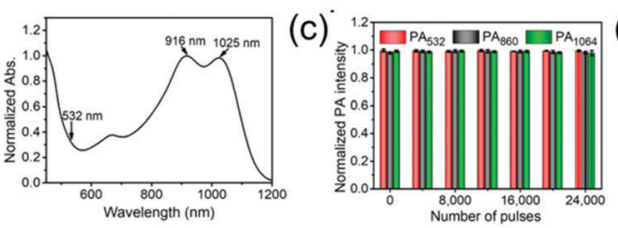

(e)
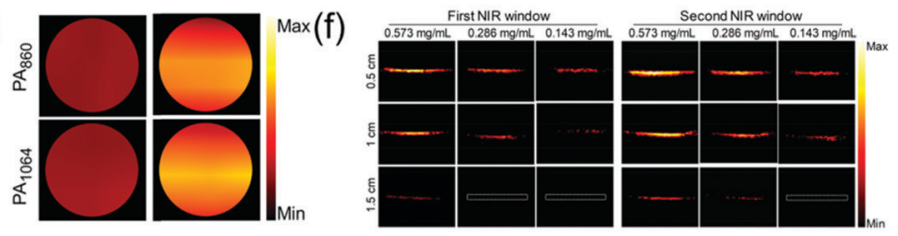

(g)
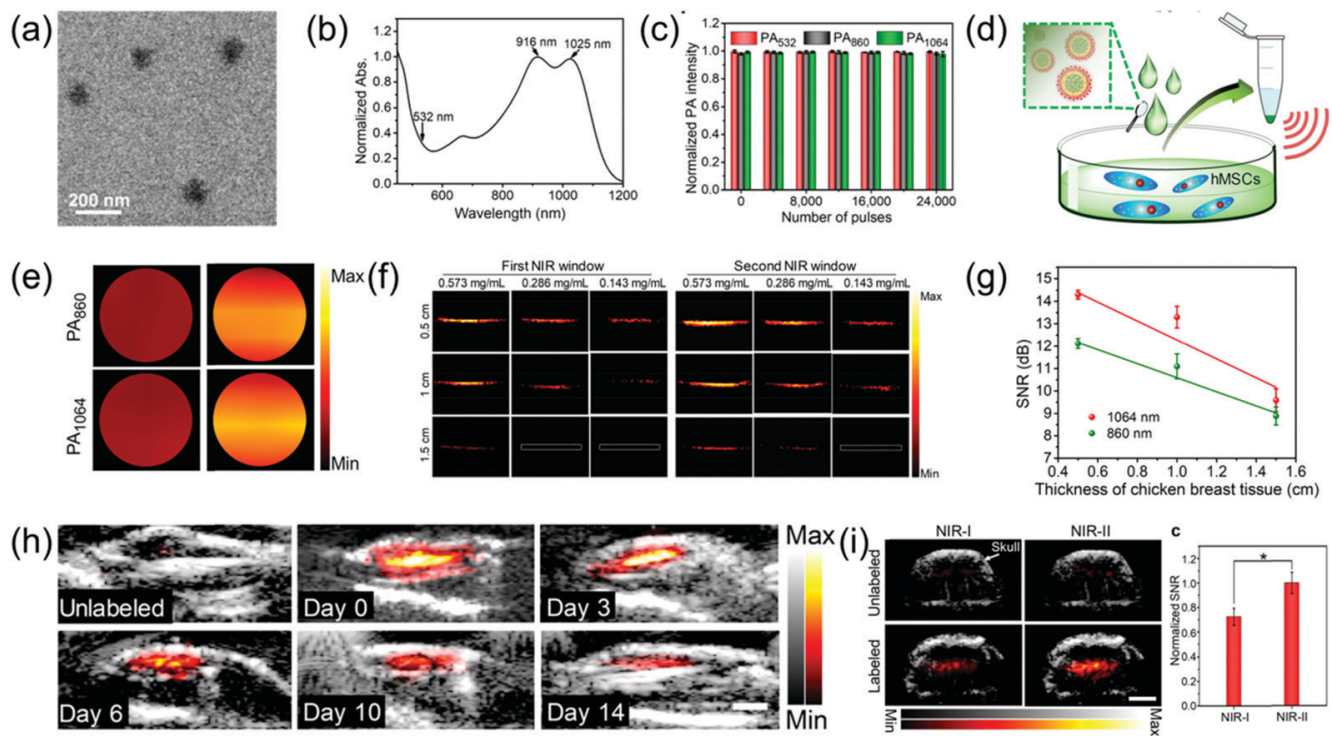

Fig. 9 (a) TEM image of OSPNs ${ }^{+}$nanoparticles, (b) absorption spectra of OSPNs ${ }^{+}$, (c) PA signal intensity of the OSPNs ${ }^{+}$under laser excitation at different wavelengths, (d) proposed mechanism of the hMSCs incubated with OSPNs ${ }^{+}$, followed by concentration for PA imaging, (e) PAI of the collected hMSCs treated by OSPNs ${ }^{+}$or OSPNs ${ }^{-}$, (f) comparison of PAI in the NIR-I and NIR-II windows at different imaging depths, (g) SNR of PAI as a function of PA imaging depth, (h) combined ultrasound and PAl of subcutaneously transplanted unlabeled or OSPNs ${ }^{+}$-labeled hMSCs, and (i) merged ultrasound and PAI and SNR of brain transplanted unlabeled or OSPNs ${ }^{+}$-labeled hMSCs. (a-i) were reproduced from ref. 67 with permission from the American Chemical Society, Copyright 2018. 
attention due to its merits of deep penetration ability, high MPE, and excellent ablation of tumor.

\section{Comparison of photothermal therapy effects between first and second near-infrared windows}

NIR-II PTT has attracted increasing attention due to merits of deep tissue penetration and high MPE. For example, Duan et al. developed monodispersed and compact Au plasmonic blackbodies (AuPBs) with a hyperbranched structure via a one-pot, seedless, and surfactant-free method using mussel-inspired dopamine (Fig. 10a). ${ }^{16}$ The plasmonic coupling between $\mathrm{Au}$ branches led to a uniform and strong absorption from $400 \mathrm{~nm}$ to $1350 \mathrm{~nm}$. The absorption of AuPBs provided more than $80 \%$ photothermal efficiency and a closely matched photothermal ability in both the NIR-II and NIR-I windows, indicating it is an excellent contrast agent for PA imaging-guided PTT. Several in vitro and in vivo studies confirmed that the most remarkable benefit of NIR-II imaging-guided PTT is the higher MPE of the NIR-II laser (Fig. 10b-g). A subcutaneous 4T1 mouse tumor model was used to evaluate the PTT ability. Tumor-bearing mice were covered with $5 \mathrm{~mm}$ of chicken breast tissue followed by injection of $100 \mu \mathrm{L}$ of $100 \mu \mathrm{L} \mathrm{mL}^{-1}$ an AuPB probe. The mice were then exposed to light illumination of a MPE dose (Fig. 10d). As shown in Fig. 10e, only the tumor irradiated with the $1064 \mathrm{~nm}$ laser at AuPBs post-injection showed tumor growth, while the tumor irradiated with the $808 \mathrm{~nm}$ laser (a)
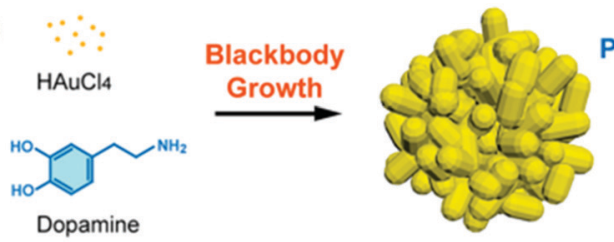

(c)

(b)

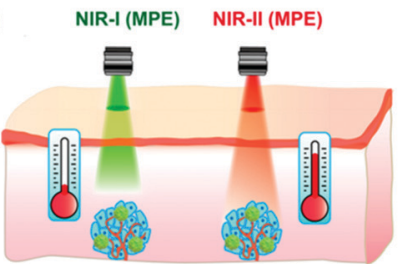

(e)

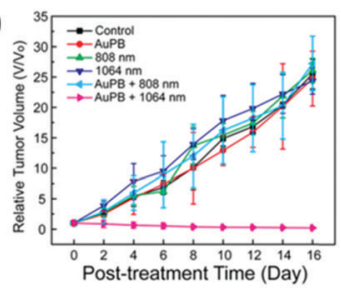

)

(f)

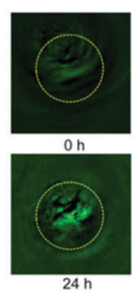

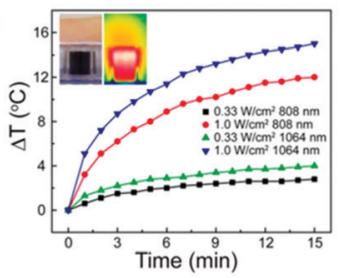

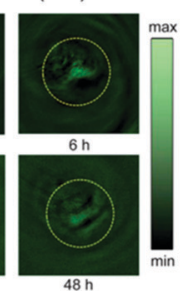

(h)

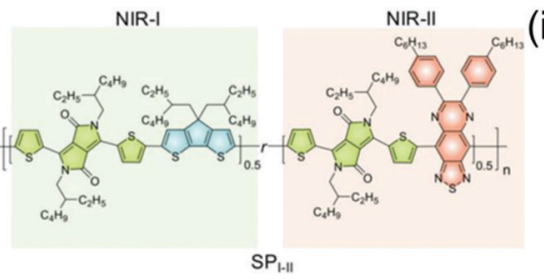

(k)

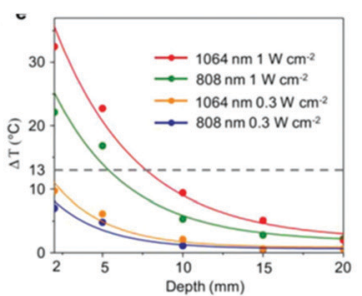

(l)

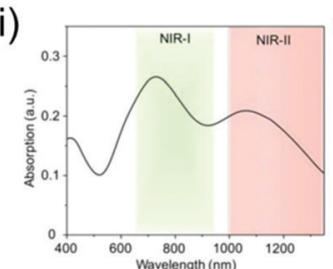

(m) (d)

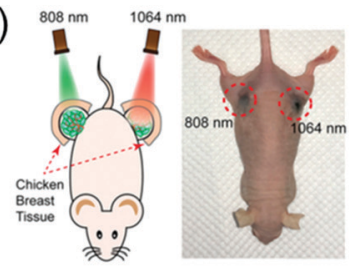

(g)

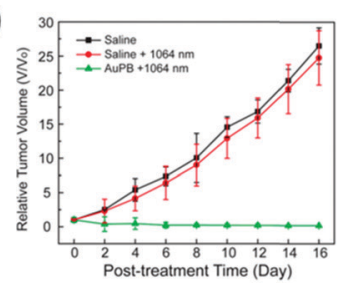

(j)

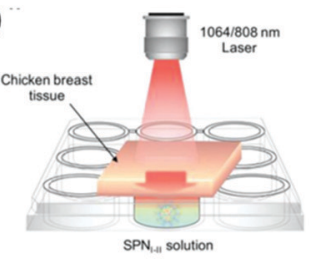

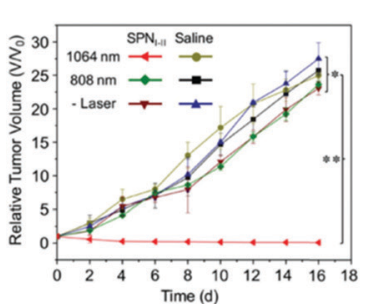

Fig. 10 (a) Proposed mechanism of the synthesis of AuPBs, (b) comparative photothermal therapy of AuPBs under MPE of NIR-I and NIR-II illumination, (c) photothermal performance of $200 \mu \mathrm{g} \mathrm{mL}^{-1}$ AuPB dispersion insulated by chicken breast tissue of $10 \mathrm{~mm}$ thickness, (d) proposed mechanism of in vivo PTT on tumor-bearing mice covered by $0.5 \mathrm{~cm}$ of tissue after laser irradiation, (e) data of different groups of mice after treatment, (f) in vivo PAl of tumor region at $1064 \mathrm{~nm}$ irradiation at different time points, (g) relative tumor volume, (h) chemical structure of SPI-II, (i) absorption spectra of SPNI-II, (j) proposed mechanism of deep-tissue photothermal study, (k) temperature change of $50 \mu \mathrm{g} \mathrm{mL}^{-1} \mathrm{SPNI}$-II solution at different depths of chicken breast tissue via different laser irradiations, (l) proposed mechanism of deep tissue PTT and $(\mathrm{m})$ tumor growth curves of mice after treatment at different conditions. (a-g) were reproduced from ref. 16 with permission from the American Chemical Society, Copyright 2018 and ( $h-m)$ were reproduced from ref. 85 with permission from Wiley-VCH, Copyright 2018. 
showed no obvious therapeutic effect. This result shows that the efficacy of NIR-II-mediated PTT is superior to that of NIR-Imediated PTT. In addition, the accumulation of AuPBs at the tumor site via intravenous injection led to a 4.7-fold higher PA intensity in the tumor region compared to that of the tumor background at $24 \mathrm{~h}$ post-injection (Fig. 10f). Fig. $10 \mathrm{~g}$ shows that PA imaging-guided NIR-II PTT achieved an extremely significant therapeutic effect. To investigate the superiority of the NIR-II laser over NIR-I laser in terms of tumor PTT, Pu et al. developed a semiconducting copolymer $\mathrm{SPN}_{\mathrm{I}-\mathrm{II}}$ with wide-band absorption in both NIR-I and NIR-II windows via the nanoprecipitation method with PEG- $b$-PPG- $b$-PEG (Fig. $10 \mathrm{~h}$ and i). ${ }^{85}$ To compare the PTT abilities between the NIR-I and NIR-II windows, $\mathrm{SPN}_{\mathrm{I}-\mathrm{II}}$ was buried in chicken breast tissue of different thicknesses followed by irradiation at 808 or $1064 \mathrm{~nm}$ within the permitted laser power density (Fig. 10j). The temperature variation of $\mathrm{SPN}_{\mathrm{I}-\mathrm{II}}$ illuminated at $1064 \mathrm{~nm}$ was significantly superior to that illuminated at $808 \mathrm{~nm}$ at the same tissue depths at their respective MPE limit, respectively (Fig. 10k). The deep-tissue tumor model that was covered with $5 \mathrm{~mm}$ chicken breast tissue, intratumorally injected $\mathrm{SPN}_{\mathrm{I}-\mathrm{II}}$, and treated with $1064 \mathrm{~nm}$ laser illumination produced higher heat and wholly ablated tumors, while the $808 \mathrm{~nm}$ laser did not cause these results (Fig. 10l and m).
In summary, the effect of PTT in the NIR-II window is much better than that of the NIR-I window.

\section{Photoacoustic imaging-guided second near-infrared window photothermal therapy}

NIR light-induced PTT has received increasing attention in recent years. ${ }^{86-88}$ The tissue penetration depth of the NIR-II window has been identified to be stronger than that of the NIR-I window. Recently, imaging-guided second near-infrared window photothermal therapy has been strongly developed. ${ }^{26,47,48,52,69,89-92}$ For example, Cheng et al. grafted gold onto the surface of $\mathrm{Ti}_{3} \mathrm{C}_{2}$ nanosheets, thus effectively improving its light absorption in both the NIR-I and NIR-II windows (Fig. 11a). ${ }^{73}$ The as-prepared $\mathrm{Ti}_{3} \mathrm{C}_{2} @ A u$ nanocomposites with enhanced optical absorption in the NIR-II window and excellent X-ray attenuation capabilities were used as nanoagents for PA and CT dual-modal imagingguided NIR-II PTT. Furthermore, the mild PT effect of the $\mathrm{Ti}_{3} \mathrm{C}_{2} @ \mathrm{Au}$ nanocomposites in the NIR-II region could improve tumor oxygenation, which remarkedly increased radiotherapy (Fig. 11b-d). Li et al. reported that peptide (LyP-1)-labeled bismuth (Bi) nanoparticles (Bi-LyP-1 NPs) exhibited high tumor accumulation and cellular uptake abilities as well as enhanced absorption in the NIR-II window. Therefore, they were employed (a)

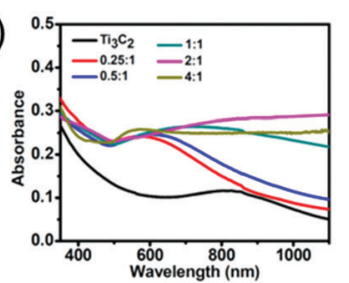

(d)

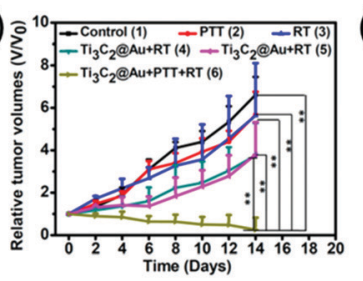

(g)

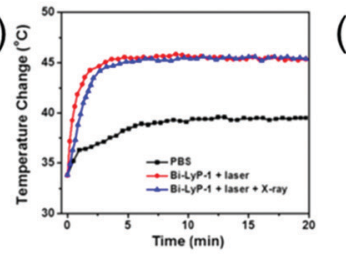

(h)

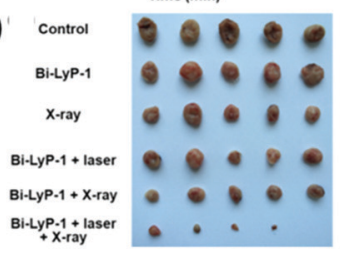

(b)
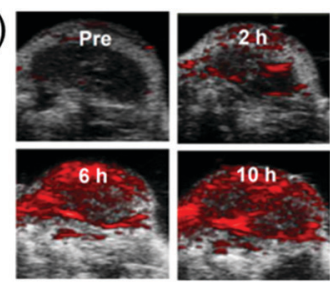

(e)

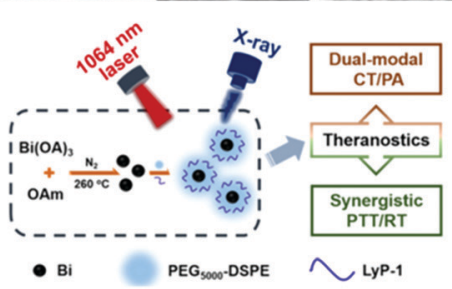

(i)

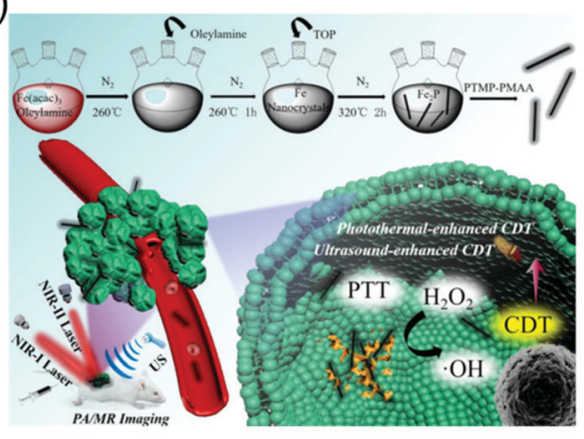

(c)

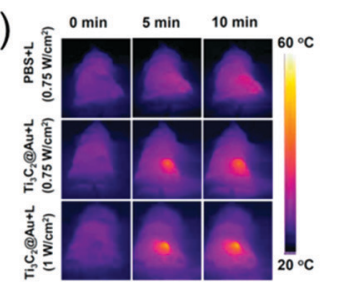

(f)

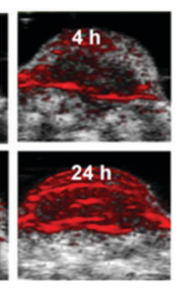

$0 \mathrm{~min}$

i.v. injection

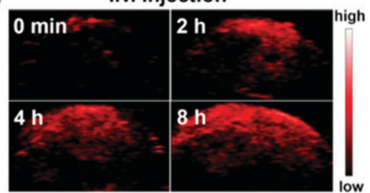

(j)

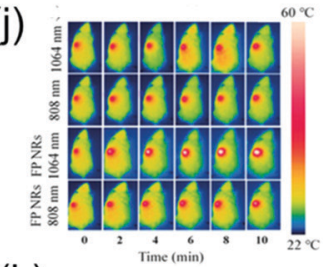

(k)

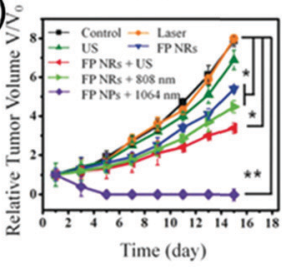

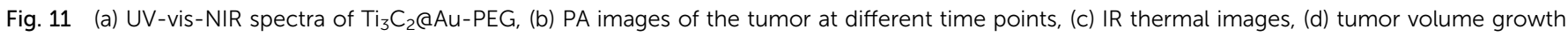

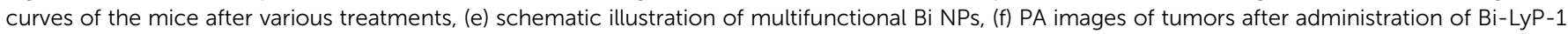

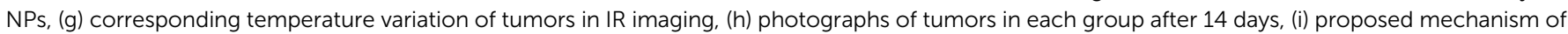

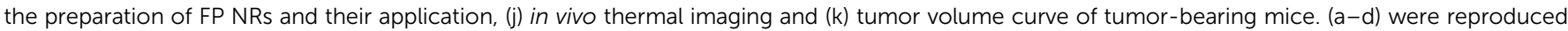

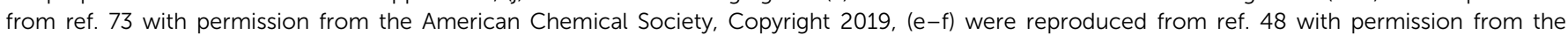
American Chemical Society, Copyright 2017 and (i-k) were reproduced from ref. 93 with permission from Wiley-VCH, Copyright 2018. 
for both dual-modal PA and CT imaging-guided synergistic PTT and tumor radiotherapy (Fig. 11e). ${ }^{48}$ The PA signal intensity at $8 \mathrm{~h}$ post-injection of Bi-LyP-1 NPs was about 3.6-fold higher than that before injection (Fig. 11f), suggesting Bi nanoparticles are excellent contrast agents with great potential for PA imaging. For NIR-II PTT and radiotherapy in vivo, tumor-bearing mice were intravenously injected with $200 \mu \mathrm{L}$ of $3 \mathrm{mg} \mathrm{mL}^{-1}$ of Bi-LyP-1 NPs and the mice were then irradiated with a $1064 \mathrm{~nm}$ laser at $0.6 \mathrm{~W} \mathrm{~cm} \mathrm{~cm}^{-2}$. Consequently, the temperature of the tumors instantly increased to $45{ }^{\circ} \mathrm{C}$ (Fig. 11g), indicating the Bi-LyP-1 NPs are promising PT agents with positive synergistic PTT and radiotherapy effects on inhibiting tumor growth (Fig. 11h). Liu et al. developed ferrous phosphide nanorods (FP NRs) with a photothermal conversion efficiency of up to $56.6 \%$ and ultrasound- and PT-enhanced Fenton reaction properties in the NIR-II window, which exhibited significant synergistic therapeutic abilities. In addition, the FP NRs could be employed as promising PAI and magnetic resonance imaging agents due to their excellent photothermal conversion efficiency and high traverse relaxivity (Fig. 11i). Therefore, FP NPs were used as diagnostic agents for synergetic PTT and PT-enhanced chemodynamic therapy (Fig. $11 \mathrm{j}$ and k). ${ }^{93}$

\section{NIR-II photoacoustic imaging-guided NIR-II window photothermal therapy}

Presently, there are very few reports of NIR-II PA imagingguided PTT in the NIR-II window due to the lack of desirable nanomaterials and advanced NIR-II window PA imaging equipment. Very recently, Chen et al. developed a multifunctional theranostic composite nanocatalyst by conjugating $\mathrm{Fe}_{3} \mathrm{O}_{4}$ modified polypyrrole (PPy) nanoparticles with glucose oxidase (GOD) to achieve NIR-II PA/MR imaging-guided photothermal and photothermal-enhanced cancer-specific sequential nanocatalytic chemodynamic combination therapy (Fig. 12a), ${ }^{84}$ which illuminates the path of NIR-II PA imaging-guided NIR-II PTT. The nanoagent $\left(\mathrm{Fe}_{3} \mathrm{O}_{4} @ P P y @ G O D\right)$ with a broadband absorption ranging from the NIR-I to NIR-II region and excellent magnetic properties severed as contrast agents for MR and PA imaging in both NIR-I and NIR-II windows. As shown in Fig. $5 \mathrm{~b}$ and $\mathrm{c}$, in vitro PA and MR imaging exhibited a linear relationship between concentration of sample and signal intensities. The in vivo assay showed the PA intensities at NIR-I or NIR-II window in the tumor site were enhanced gradually with time post-injection of the nanoagent, implying the time-dependent distribution of the $\mathrm{Fe}_{3} \mathrm{O}_{4} @ P P y @ G O D$ nanoagent in the tumor site. In addition, before injection, the signal in the NIR-I window in the tumor site was stronger than that in the NIR-II window because the absorption of blood in the NIR-II window was minimal (Fig. 5b). NIR-II PA imaging combined with other molecular imaging techniques forms multi-modal molecular imaging, which overcomes the shortcomings of a single imaging mode, realizes complementary advantages, and widens the application range of molecular imaging technology. Herein, MR imaging showed that the intravenous injection of $\mathrm{Fe}_{3} \mathrm{O}_{4} @ P P y @ G O D$ nanoagents could achieve a more uniform distribution in the whole tumor region compared to intratumoral injection (Fig. 5c). Encouraged by the good imaging quality, PTT in both NIR-I and NIR-II windows was performed, which showed a better treatment effect with NIR-II laser irradiation (Fig. 5d-f).

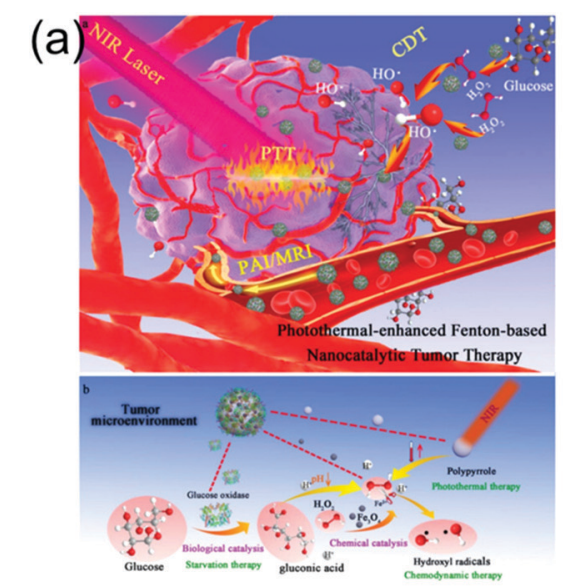

(d)

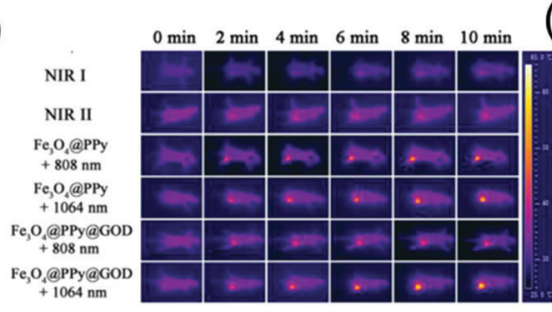

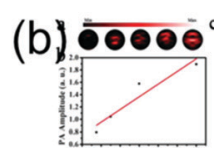
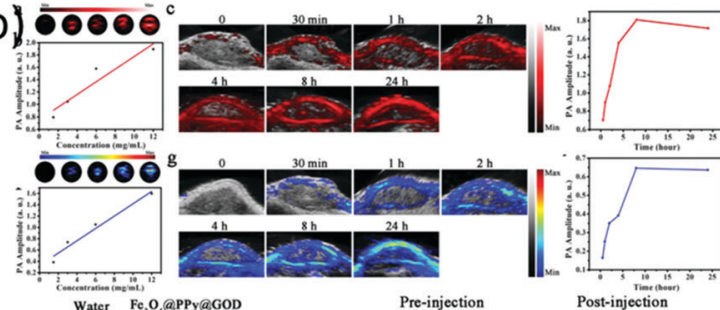

(C)

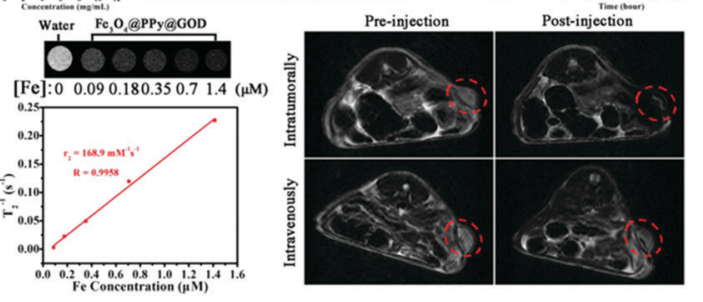

$(\mathrm{e})$

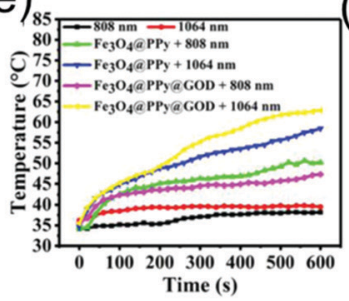

(f)

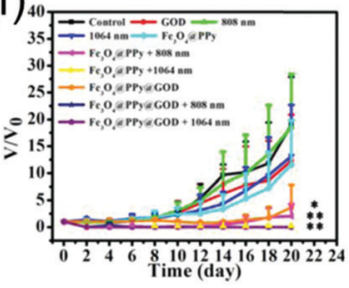

Fig. 12 (a) Schematic illustration of NIR-II PA/MR imaging-guided NIR-II PTT combination PTT, (b) PA imaging in both NIR-I and NIR-II windows, (c) MR imaging in vitro and vivo, (d) IR thermal images and (e) corresponding temperature changes and (f) time-dependent relative tumor volume. (a-f) were reproduced from ref. 84 with permission from Wiley-VCH, Copyright 2018. 


\section{Prospects and challenges}

The latest bioimaging techniques indicate that the NIR-II laser has become a promising alternative to the more commonly used NIR-I window. It has been reported that biological tissues such as deoxyhemoglobin, oxyhemoglobin, skin, and fat have lower extinction coefficients in the NIR-II window, resulting in a significant reduction in the interaction between these tissue components and longer wavelength photons. Consequently, the absorption and scattering of light in the NIR-II region can be significantly reduced. In addition, PTT using NIR-II light with longer wavelengths exhibit enhanced SBR, high MPE and highly efficient ablation of solid tumors, and exerts minimal side-effects for normal tissue. Encouraged by the numerous merits of NIR-II light, recently, NIR-II PAI and imaging-guided NIR-II PTT have been rapidly developed.

Although PAI and PTT offer significant advantages in the NIR-II window, considerable work is still necessary to meet future challenges in the field of biomedicine. Firstly, designing NIR-II PAI contrast agents or PT agents for their further application in fundamental and clinical research requires further development. Secondly, overcoming biological safety problems such as toxicity, biocompatibility, and pharmacokinetics of NIR-II nanoagents is a top priority. Finally, both the further development and optimization of NIR-II PAI and PT instruments are required for the improvement of imaging quality and photothermal effect.

In summary, NIR-II PAI and PTT offer pronounced prospects for development; however, improving their imaging quality and PT effect as well as broadening their application scope to meet the requirements of life sciences and medical research still remains urgent issues. It has been suggested that with the continuous progress of nanoscience and the rapid development of advanced instruments, these problems will be resolved in the near future.

\section{Conflicts of interest}

There are no conflicts to declare.

\section{Acknowledgements}

This research was supported by National Natural Science Foundation of China (NSFC) projects (Grant number: 81871384, 81771869, 81571708, 81501506 and 21874024), the Research Fund of Science and Technology Department of Jilin Province (Grant number: 20160101001JC), the Norman Bethune Program of Jilin University (Grant number: 2015219) and the Hygiene Specific Subjects of Jilin Province (Grant number: 2018SCZ039).

\section{Notes and references}

1 Q. Fu, R. Zhu, J. Song, H. Yang and X. Chen, Adv. Mater., 2019, 31, 1805875.

2 K. S. Valluru and J. K. Willmann, Ultrasonography, 2016, 35, 267-280.
3 S. Wang, J. Lin, T. Wang, X. Chen and P. Huang, Theranostics, 2016, 6, 2394-2413.

4 Y. Jiang and K. Pu, Small, 2017, 13, 1700710.

5 X. L. Dean-Ben, S. Gottschalk, B. Mc Larney, S. Shoham and

D. Razansky, Chem. Soc. Rev., 2017, 46, 2158-2198.

6 X. Huang, J. Song, B. C. Yung, X. Huang, Y. Xiong and X. Chen, Chem. Soc. Rev., 2018, 47, 2873-2920.

7 L. V. Wang and S. Hu, Science, 2012, 335, 1458.

8 L. Nie and X. Chen, Chem. Soc. Rev., 2014, 43, 7132-7170.

9 S. Zackrisson, S. M. W. Y. van de Ven and S. S. Gambhir, Cancer Res., 2014, 74, 979-1004.

10 W. Li and X. Chen, Nanomedicine, 2015, 10, 299-320.

11 A. Taruttis and V. Ntziachristos, Nat. Photonics, 2015, 9, 219-227.

12 L. V. Wang and J. Yao, Nat. Methods, 2016, 13, 627-638.

13 J. Weber, P. C. Beard and S. E. Bohndiek, Nat. Methods, 2016, 13, 639-650.

14 C. J. Reinhardt and J. Chan, Biochemistry, 2018, 57, 194-199. $15 \mathrm{~J} . \mathrm{Wu}, \mathrm{L}$. You, L. Lan, H. J. Lee, S. T. Chaudhry, R. Li, J. X. Cheng and J. Mei, Adv. Mater., 2017, 29, 1703403.

16 J. Zhou, Y. Jiang, S. Hou, P. K. Upputuri, D. Wu, J. Li, P. Wang, X. Zhen, M. Pramanik, K. Pu and H. Duan, ACS Nano, 2018, 12, 2643-2651.

17 G. Hong, A. L. Antaris and H. Dai, Nat. Biomed. Eng., 2017, 1, 0010 .

18 A. M. Smith, M. C. Mancini and S. Nie, Nat. Nanotechnol., 2009, 4, 710-711.

19 A. N. Bashkatov, E. A. Genina, V. I. Kochubey and V. V. Tuchin, J. Phys. D: Appl. Phys., 2005, 38, 2543-2555.

20 Y. Cao, J.-H. Dou, N.-j. Zhao, S. Zhang, Y.-Q. Zheng, J.-P. Zhang, J.-Y. Wang, J. Pei and Y. Wang, Chem. Mater., 2016, 29, 718-725.

21 K. Homan, S. Kim, Y.-S. Chen, B. Wang, S. Mallidi and S. Emelianov, Opt. Lett., 2010, 35, 2663-2665.

22 Y. S. Chen, Y. Zhao, S. J. Yoon, S. S. Gambhir and S. Emelianov, Nat. Nanotechnol., DOI: 10.1038/s41565-019-0392-3.

23 G. Ku, M. Zhou, S. Song, Q. Huang, J. Hazle and C. Li, ACS Nano, 2012, 6, 7489-7496.

24 A. S. Hannah, D. VanderLaan, Y. S. Chen and S. Y. Emelianov, Biomed. Opt. Express, 2014, 5, 3042-3052.

25 Y. Zhou, D. Wang, Y. Zhang, U. Chitgupi, J. Geng, Y. Wang, Y. Zhang, T. R. Cook, J. Xia and J. F. Lovell, Theranostics, 2016, 6, 688-697.

26 Z. Cao, L. Feng, G. Zhang, J. Wang, S. Shen, D. Li and X. Yang, Biomaterials, 2018, 155, 103-111.

27 J. F. Lovell, T. W. B. Liu, J. Chen and G. Zheng, Chem. Rev., 2010, 110, 2839-2857.

28 J. U. Menon, P. Jadeja, P. Tambe, V. Khanh, B. Yuan and K. T. Nguyen, Theranostics, 2013, 3, 152-166.

29 Q. Dong, X. Wang, X. Hu, L. Xiao, L. Zhang, L. Song, M. Xu, Y. Zou, L. Chen, Z. Chen and W. Tan, Angew. Chem., Int. Ed., 2018, 57, 177-181.

30 H. Wang, J. Chang, M. Shi, W. Pan, N. Li and B. Tang, Angew. Chem., Int. Ed., 2019, 58, 1057-1061.

31 X. Wang, Y. Ma, X. Sheng, Y. Wang and H. Xu, Nano Lett., 2018, 18, 2217-2225. 
32 S. Li, L. Zhang, X. Chen, T. Wang, Y. Zhao, L. Li and C. Wang, ACS Appl. Mater. Interfaces, 2018, 10, 24137-24148.

33 L. Zhang, S. Li, X. Chen, T. Wang, L. Li, Z. Su and C. Wang, Adv. Funct. Mater., 2018, 28, 1803815.

34 X. Zhen, J. Zhang, J. Huang, C. Xie, Q. Miao and K. Pu, Angew. Chem., Int. Ed., 2018, 57, 7804-7808.

35 Q. Chen, C. Wang, Z. Zhan, W. He, Z. Cheng, Y. Li and Z. Liu, Biomaterials, 2014, 35, 8206-8214.

36 Q. Chen, C. Wang, C. Liang and Z. Liu, J. Controlled Release, 2015, 213, e89.

37 L. Li, Y. Liu, P. Hao, Z. Wang, L. Fu, Z. Ma and J. Zhou, Biomaterials, 2015, 41, 132-140.

38 Y. Tian, J. Zhang, S. Tang, L. Zhou and W. Yang, Small, 2016, 12, 721-726.

39 M. Chu, Y. Shao, J. Peng, X. Dai, H. Li, Q. Wu and D. Shi, Biomaterials, 2013, 34, 4078-4088.

40 L.-E. Deng, Y. Li, L. Gong and J. Wang, J. Inorg. Mater., 2018, 33, 825-831.

41 Y. Cheng, Y. Chang, Y. Feng, H. Jian, Z. Tang and H. Zhang, Angew. Chem., Int. Ed., 2018, 57, 246-251.

42 Y. Zhang, F. Huang, C. Ren, J. Liu, L. Yang, S. Chen, J. Chang, C. Yang, W. Wang, C. Zhang, Q. Liu, X.-J. Liang and J. Liu, Adv. Sci., 2019, 1801806.

43 R. Chen, J. Wang and H. Qiao, Prog. Chem., 2017, 29, 329-336.

44 H. S. Jung, P. Verwilst, A. Sharma, J. Shin, J. L. Sessler and J. S. Kim, Chem. Soc. Rev., 2018, 47, 2280-2297.

45 Y. Liu, P. Bhattarai, Z. Dai and X. Chen, Chem. Soc. Rev., DOI: $10.1039 / \mathrm{C} 8 \mathrm{CS} 00618 \mathrm{~K}$.

46 M.-F. Tsai, S.-H. G. Chang, F.-Y. Cheng, V. Shanmugam, Y.-S. Cheng, C.-H. Su and C.-S. Yeh, ACS Nano, 2013, 7, 5330-5342.

47 K. Cai, W. Zhang, J. Zhang, H. Li, H. Han and T. Zhai, ACS Appl. Mater. Interfaces, 2018, 10, 36703-36710.

48 X. Yu, A. Li, C. Zhao, K. Yang, X. Chen and W. Li, ACS Nano, 2017, 11, 3990-4001.

49 S. Park, G. Park, J. Kim, W. Choi, U. Jeong and C. Kim, Nanoscale, 2018, 10, 20548-20558.

50 H. Yan, J. Chen, Y. Li, Y. Bai, Y. Wu, Z. Sheng, L. Song, C. Liu and H. Zhang, Biomater. Sci., 2018, 7, 92-103.

51 A. Li, X. Li, X. Yu, W. Li, R. Zhao, X. An, D. Cui, X. Chen and W. Li, Biomaterials, 2017, 112, 164-175.

52 K. Ke, W. Yang, X. Xie, R. Liu, L. L. Wang, W. W. Lin, G. Huang, C. H. Lu and H. H. Yang, Theranostics, 2017, 7, 4763-4776.

53 L. Zhou, Y. Jing, Y. Liu, Z. Liu, D. Gao, H. Chen, W. Song, T. Wang, X. Fang, W. Qin, Z. Yuan, S. Dai, Z. A. Qiao and C. Wu, Theranostics, 2018, 8, 663-675.

54 X. Yu, K. Yang, X. Chen and W. Li, Biomaterials, 2017, 143, 120-129.

55 K. Pu, A. J. Shuhendler, J. V. Jokerst, J. Mei, S. S. Gambhir, Z. Bao and J. Rao, Nat. Nanotechnol., 2014, 9, 233-239.

56 J. Li, J. Rao and K. Pu, Biomaterials, 2018, 155, 217-235.

57 Y. Jiang and K. Pu, Acc. Chem. Res., 2018, 51, 1840-1849.

58 Y. Lyu and K. Pu, Adv. Sci., 2017, 4, 1600481.

59 Y. Lyu, J. Zeng, Y. Jiang, X. Zhen, T. Wang, S. Qiu, X. Lou, M. Gao and K. Pu, ACS Nano, 2018, 12, 1801-1810.
60 C. Xie, X. Zhen, Y. Lyu and K. Pu, Adv. Mater., 2017, 29, 1703693.

61 X. Zhen, C. Xie, Y. Jiang, X. Ai, B. Xing and K. Pu, Nano Lett., 2018, 18, 1498-1505.

62 Y. Jiang, P. K. Upputuri, C. Xie, Y. Lyu, L. Zhang, Q. Xiong, M. Pramanik and K. Pu, Nano Lett., 2017, 17, 4964-4969.

63 Y. Jiang, P. K. Upputuri, C. Xie, Z. Zeng, A. Sharma, X. Zhen, J. Li, J. Huang, M. Pramanik and K. Pu, Adv. Mater., 2019, e1808166.

64 Y. Lyu and K. Pu, Adv. Sci., 2017, 4, 1600481.

65 B. Guo, Z. Sheng, K. Kenry, D. Hu, X. Lin, S. Xu, C. Liu, H. Zheng and B. Liu, Mater. Horiz., 2017, 4, 1151-1156.

66 B. Guo, Z. Sheng, D. Hu, C. Liu, H. Zheng and B. Liu, Adv. Mater., 2018, 30, 1802591.

67 C. Yin, G. Wen, C. Liu, B. Yang, S. Lin, J. Huang, P. Zhao, S. H. D. Wong, K. Zhang, X. Chen, G. Li, X. Jiang, J. Huang, K. Pu, L. Wang and L. Bian, ACS Nano, 2018, 12, 12201-12211.

68 P. K. Upputuri, C. Yang, S. Huang, K. Wang, M. Wang and M. Pramanik, J. Biomed. Opt., 2018, 24, 1-7.

69 Y. Cao, T. Wu, K. Zhang, X. Meng, W. Dai, D. Wang, H. Dong and X. Zhang, ACS Nano, 2019, 13, 1499-1510.

70 N. Yu, Y. Hu, X. Wang, G. Liu, Z. Wang, Z. Liu, Q. Tian, M. Zhu, X. Shi and Z. Chen, Nanoscale, 2017, 9, 9148-9159.

71 C. Guo, S. Yin, Q. Dong and T. Sato, Nanoscale, 2012, 4, 3394-3398.

72 Y. Cheng, F. Yang, G. Xiang, K. Zhang, Y. Cao, D. Wang, H. Dong and X. Zhang, Nano Lett., 2019, 19, 1179-1189.

73 W. Tang, Z. Dong, R. Zhang, X. Yi, K. Yang, M. Jin, C. Yuan, Z. Xiao, Z. Liu and L. Cheng, ACS Nano, 2018, 13, 284-294.

74 K. Huang, Y. Zhang, J. Lin and P. Huang, Biomater. Sci., 2019, 7, 472-479.

75 Y. Jiang and K. Pu, Adv. Biosyst., 2018, 2, 1700262.

76 Y. Liu, D. Gao, M. Xu and Z. Yuan, J. Biophotonics, 2018, e201800237.

77 X. Meng, J. Zhang, Z. Sun, L. Zhou, G. Deng, S. Li, W. Li, P. Gong and L. Cai, Theranostics, 2018, 8, 6025-6034.

78 T. Bohn, S. Rapp, N. Luther, M. Klein, T. J. Bruehl, N. Kojima, P. Aranda Lopez, J. Hahlbrock, S. Muth, S. Endo, S. Pektor, A. Brand, K. Renner, V. Popp, K. Gerlach, D. Vogel, C. Lueckel, D. Arnold-Schild, J. Pouyssegur, M. Kreutz, M. Huber, J. Koenig, B. Weigmann, H. C. Probst, E. von Stebut, C. Becker, H. Schild, E. Schmitt and T. Bopp, Nat. Immunol., 2018, 19, 1319-1329.

79 W. Xie, Q. Gao, D. Wang, Z. Guo, F. Gao, X. Wang, Q. Cai, S.-s. Feng, H. Fan, X. Sun and L. Zhao, Nano Res., 2018, 11, 2470-2487.

80 M. Abbas, Q. Zou, S. Li and X. Yan, Adv. Mater., 2017, 29, 1605021.

81 R. Vankayala and K. C. Hwang, Adv. Mater., 2018, 30, 1706320.

82 J.-J. Hu, Y.-J. Cheng and X.-Z. Zhang, Nanoscale, 2018, 10, 22657-22672.

83 B. Shi, Q. Yan, J. Tang, K. Xin, J. Zhang, Y. Zhu, G. Xu, R. Wang, J. Chen, W. Gao, T. Zhu, J. Shi, C. Fan, C. Zhao and H. Tian, Nano Lett., 2018, 18, 6411-6416.

84 W. Feng, X. Han, R. Wang, X. Gao, P. Hu, W. Yue, Y. Chen and J. Shi, Adv. Mater., 2019, 31, 1805919. 
85 Y. Jiang, J. Li, X. Zhen, C. Xie and K. Pu, Adv. Mater., 2018, 30, e1705980.

86 H. Lin, S. Gao, C. Dai, Y. Chen and J. Shi, J. Am. Chem. Soc., 2017, 139, 16235-16247.

87 P. Zhu, S. Gao, H. Lin, X. Lu, B. Yang, L. Zhang, Y. Chen and J. Shi, Nano Lett., 2019, 19, 2128-2137.

88 T. Li, C. Li, Z. Ruan, P. Xu, X. Yang, P. Yuan, Q. Wang and L. Yan, ACS Nano, 2019, 13, 3691-3702.

89 T. Sun, J. H. Dou, S. Liu, X. Wang, X. Zheng, Y. Wang, J. Pei and Z. Xie, ACS Appl. Mater. Interfaces, 2018, 10, 7919-7926.
90 D. Zhang, H. Xu, X. Zhang, Y. Liu, M. Wu, J. Li, H. Yang, G. Liu, X. Liu, J. Liu and Z. Yuan, ACS Appl. Mater. Interfaces, 2018, 10, 25203-25212.

91 P. Vijayaraghavan, C.-H. Liu, R. Vankayala, C.-S. Chiang and K. C. Hwang, Adv. Mater., 2014, 26, 6689-6695.

92 Q. Ma, L. Cheng, F. Gong, Z. Dong, C. Liang, M. Wang, L. Feng, Y. Li, Z. Liu, C. Li and L. He, J. Mater. Chem. B, 2018, 6, 5069-5079.

93 Y. Liu, W. Zhen, Y. Wang, J. Liu, L. Jin, T. Zhang, S. Zhang, Y. Zhao, S. Song, C. Li, J. Zhu, Y. Yang and H. Zhang, Angew. Chem., Int. Ed., 2019, 58, 2407-2412. 